\title{
A falling magnetic monopole as a holographic local quench
}

\author{
Nicolò Zenoni, ${ }^{a, b, c}$ Roberto Auzzi, ${ }^{a, b}$ Stefania Caggioli, ${ }^{a}$ Maria Martinelli ${ }^{a}$ and \\ Giuseppe Nardelli ${ }^{a, d}$ \\ ${ }^{a}$ Dipartimento di Matematica e Fisica, Università Cattolica del Sacro Cuore, \\ Via Musei 41, 25121 Brescia, Italy \\ ${ }^{b}$ INFN Sezione di Perugia, \\ Via A. Pascoli, 06123 Perugia, Italy \\ ${ }^{c}$ Instituut voor Theoretische Fysica, KU Leuven, \\ Celestijnenlaan 200D, B-3001 Leuven, Belgium \\ ${ }^{d}$ TIFPA - INFN, c/o Dipartimento di Fisica, Università di Trento, \\ 38123 Povo (TN), Italy \\ E-mail: nicolo.zenoni@unicatt.it, roberto.auzzi@unicatt.it, \\ stefy.caggioli@gmail.com, maria.martinelli1996@gmail.com, \\ giuseppe.nardelli@unicatt.it
}

Abstract: An analytic static monopole solution is found in global $\mathrm{AdS}_{4}$, in the limit of small backreaction. This solution is mapped in Poincaré patch to a falling monopole configuration, which is dual to a local quench triggered by the injection of a condensate. Choosing boundary conditions which are dual to a time-independent Hamiltonian, we find the same functional form of the energy-momentum tensor as the one of a quench dual to a falling black hole. On the contrary, the details of the spread of entanglement entropy are very different from the falling black hole case, where the quench induces always a higher entropy compared to the vacuum, i.e. $\Delta S>0$. In the propagation of entanglement entropy for the monopole quench, there is instead a competition between a negative contribution to $\Delta S$ due to the scalar condensate and a positive one carried by the freely propagating quasiparticles generated by the energy injection.

KEywords: AdS-CFT Correspondence, Solitons Monopoles and Instantons

ArXiv EPrint: 2106.13757 


\section{Contents}

1 Introduction 1

2 A static monopole in global AdS 3

2.1 Monopole solution in the probe limit 4

2.2 Monopole backreaction 5

3 A falling monopole in Poincaré patch $\quad 7$

3.1 Bulk energy density of the falling monopole 8

4 Expectation values of local operators $\quad 9$

$\begin{array}{lll}4.1 & \text { Boundary conditions for the scalar } & 10\end{array}$

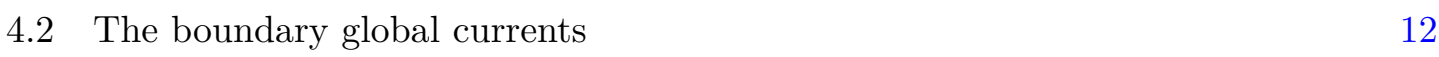

4.3 The boundary energy-momentum tensor 13

5 Holographic entanglement entropy $\quad 15$

$\begin{array}{ll}5.1 \text { Disk centered at the origin } & 16\end{array}$

$\begin{array}{ll}5.2 \text { Translated disk } & 19\end{array}$

$\begin{array}{lll}5.3 & \text { Half-plane region } & 20\end{array}$

5.4 Comparison with the BH quench 21

6 Conclusions 23

A Equations of motion $\quad 24$

A.1 Coordinate $r \quad 24$

$\begin{array}{lll}\text { A.2 Coordinate } \psi & 25\end{array}$

B Abelian field strength and flux $\quad 25$

C Details of the boundary energy-momentum tensor 26

$\begin{array}{ll}\text { C.1 Black hole } & 26\end{array}$

C.2 Monopole with Dirichlet boundary conditions 27

C.3 Monopole with Neumann and multitrace boundary conditions 28

D Details of the entanglement entropy calculations 29

D.1 Translated-disk region $\quad 29$

$\begin{array}{lll}\text { D.2 Half-plane region } & 29\end{array}$ 


\section{Introduction}

The AdS/CFT correspondence [1-3] provides a controlled environment to study the physics of a strongly-coupled conformal field theory (CFT) on the boundary by dealing with a weakly-coupled gravitational system in the Anti-de Sitter (AdS) bulk. In particular, it gives a very useful semiclassical picture of entanglement in strongly-coupled systems [4-6], by relating the entanglement entropy of a subsystem in a CFT to the area of an extremal surface anchored at the boundary of the subregion in the AdS dual. Holographic entanglement entropy is also important to understand black holes: for example, at finite temperature and in the limit of large subregions, the entanglement entropy is dominated by the thermodynamical contribution, and the Bekenstein-Hawking entropy $[7,8]$ is recovered.

The AdS/CFT correspondence provides several setups in which to investigate the thermalisation of out of equilibrium systems. Among these, quenches are conceptually simple theoretical settings, describing the evolution of a system triggered by a sudden injection of energy or a change of coupling constants. In the case of global quenches, the perturbation is spatially homogeneous and, in the bulk, it corresponds to the formation of a black hole. The evolution of the entanglement entropy has been studied in several examples of quench protocols, both on the CFT [9] and on the gravity side [5, 10-19]. While the simplest features of the spread of entanglement can be described by a model with free quasiparticles [9], a more detailed understanding needs to take into account the role of interactions [20].

A common feature of global quenches is that the energy is injected in the whole space. Once some perturbation in the entanglement entropy is detected, there is no clear way to discriminate the point where it originated. Local quenches allow for a more transparent analysis of the spread of quantum entanglement, because the initial perturbation is localised in a finite region of space. Moreover, local quenches can be realised in condensed matter systems such as cold atoms $[21,22]$ and they may provide a setup in which entanglement entropy might be experimentally measured [23, 24].

In CFT, local quenches can be modelled by joining two initially-decoupled field theories $[25,26]$ and then evolving with a time-translation invariant Hamiltonian. Another approach in field theory is to consider excited states obtained by acting with local operators on the vacuum [27-30]. In holography, the latter kind of local quenches can be described by a free falling particle-like object in AdS [31]. The topic of local quenches was studied by many authors both on the CFT and on the gravity side: for example, mutual information was considered in [32] and finite temperature aspects were investigated in [33-35]. Bulk quantum corrections were recently studied in [36]. Local quenches obtained by splitting an initial CFT into two disconnected pieces were considered in [37].

In principle, the arbitrariness of choosing the falling particle on the gravity side corresponds to several choices of local quench protocols on the field theory side. In a local quench, it is interesting to understand which aspects of the physics are universal and which one depend on the details of the quenching protocol. This raises the question of how the choice of the falling particle in AdS affects the physics of the local quench of the boundary theory. The natural candidates which are free of singularities are black holes $(\mathrm{BH})$ or solitons. The black hole case was studied by several authors, see e.g. [31, 38, 39]. In this paper 
we will explore the possibility in which the falling particle is a soliton in $\mathrm{AdS}_{4}$, focusing on the case of the 't Hooft-Polyakov monopole [40].

Monopole solutions in global $\mathrm{AdS}_{4}$ have been considered by several authors, starting from [41, 42]. AdS monopole wall configurations have been studied in [43-46]. Holographic phase transitions for AdS monopoles have been investigated in [47-50]. In this paper, we will consider the theoretical setting introduced in [51]. In this situation, specialising to a multitrace deformation, the monopole in global AdS is dual to a theory with spontaneous symmetry breaking. A previous study of such a model was performed numerically. In this work, we find an approximate analytic solution for the winding-one monopole, which includes the first-order backreaction on the metric.

Applying the change of variables introduced in [52], we map the time-independent global $\mathrm{AdS}_{4}$ solution to a falling monopole configuration in the Poincaré patch. On the field-theory side, this is dual to a local quench in a perturbed CFT, induced by the insertion of a condensate which breaks the global symmetries of the theory. Outside the quench, the $\mathrm{SU}(2)$ global symmetry of the CFT remains unbroken.

To investigate the field theory dual of the falling monopole, we compute the expectation values of various local operators. Depending on the choice of boundary conditions for the scalar, several interpretations are possible on the CFT side. For Dirichlet or Neumann boundary conditions, the falling monopole is dual to a CFT deformed by a time-dependent source, which performs a non-zero external work on the system. For a particular choice of the multitrace deformation, given in eq. (4.14), the monopole is dual to a theory with a timeindependent Hamiltonian. In this case, the expectation value of the energy-momentum tensor has the same functional form as the one in the background of a falling black hole [31]. In other words, the energy density of the quench is not sensitive to the presence of the condensate.

To further characterise the field theory dual of the falling monopole, we perturbatively compute the entanglement entropy for spherical regions. Let us denote by $\Delta S$ the difference of entropy between the excited state and the vacuum. We find a rather different behaviour for $\Delta S$ compared to the case of the falling black hole: for the monopole quench, $\Delta S$ for a region centered at the origin is always negative, while in the $\mathrm{BH}$ case $\Delta S$ is positive. The negative sign of $\Delta S$ for the monopole quench is consistent with the expectation that the formation of bound states, which are responsible for the condensate at the core of the quench, corresponds to a decrease of the number of degrees of freedom [53].

The paper is organised as follows. In section 2 we consider a static monopole solution in global AdS and we find an analytical solution in the regime of small backreaction. In section 3 we apply the change of variables introduced in [52] to the global AdS monopole. This trick transforms the global AdS static solution to a falling monopole in the Poincaré patch, which provides the holographic dual of the local quench. In section 4 we compute the expectation value of some local operators, including the energy-momentum tensor. In section 5 we study the entanglement entropy for various subsystem geometries. We conclude in section 6 . Some technical details are discussed in appendices. 


\section{A static monopole in global AdS}

We consider the same theoretical setting as in [51] which, in global AdS, is dual to a boundary theory with a spontaneously broken $\mathrm{SU}(2)$ global symmetry. The action of the model is:

$$
S=\int d^{4} x \sqrt{-g}\left[\frac{1}{16 \pi G}(R-2 \Lambda)+\mathcal{L}_{M}\right],
$$

where $\mathcal{L}_{M}$ is the matter lagrangian

$$
\mathcal{L}_{M}=-\frac{1}{4} F_{\mu \nu}^{a} F^{a \mu \nu}-\frac{1}{2} D_{\mu} \phi^{a} D^{\mu} \phi^{a}-\frac{m_{\phi}^{2}}{2}\left(\phi^{a} \phi^{a}\right) .
$$

We choose the cosmological constant and the scalar mass as follows

$$
\Lambda=-\frac{3}{L^{2}}, \quad m_{\phi}^{2}=-\frac{2}{L^{2}},
$$

where $L$ is the AdS radius. In eq. (2.2), $F_{\mu \nu}=F_{\mu \nu}^{a} \frac{\sigma_{a}}{2}$ denotes the non-abelian field strength of the $\mathrm{SU}(2)$ gauge field $A_{\mu}=A_{\mu}^{a} \frac{\sigma_{a}}{2}$, i.e.

$$
F_{\mu \nu}^{a}=\partial_{\mu} A_{\nu}^{a}-\partial_{\nu} A_{\mu}^{a}+e \epsilon^{a b c} A_{\mu}^{b} A_{\nu}^{c},
$$

with $e$ the Yang-Mills coupling. The covariant derivative acting on the adjoint scalar is

$$
D_{\mu} \phi_{a}=\partial_{\mu} \phi_{a}+e \epsilon_{a b c} A_{\mu}^{b} \phi^{c} .
$$

The equations of motion are:

$$
\begin{array}{rlrl}
D^{\mu} F_{\mu \nu}^{a}-e \epsilon^{a b c} \phi^{b} D_{\nu} \phi^{c} & =0, & g^{\mu \nu} D_{\mu} D_{\nu} \phi^{a}-m_{\phi}^{2} \phi^{a}=0, \\
R_{\mu \nu}-\frac{1}{2} R g_{\mu \nu}+\Lambda g_{\mu \nu} & =8 \pi G T_{\mu \nu},
\end{array}
$$

where $D_{\mu}$ denotes the combination of the gravitational and $\mathrm{SU}(2)$ gauge covariant derivatives, and $T_{\mu \nu}$ is the bulk energy-momentum tensor

$$
T_{\mu \nu}=D_{\mu} \phi^{a} D_{\nu} \phi^{a}+F_{a \mu \alpha} F_{a \nu}{ }^{\alpha}+g_{\mu \nu} \mathcal{L}_{M}
$$

We first consider the monopole in a global $\mathrm{AdS}_{4}$ background, with metric

$$
d s^{2}=L^{2}\left(-\left(1+r^{2}\right) d \tau^{2}+\frac{d r^{2}}{1+r^{2}}+r^{2}\left(d \theta^{2}+\sin ^{2} \theta d \varphi^{2}\right)\right) .
$$

At large $r$ the field $\phi^{a}$ has the following expansion

$$
\phi^{a}=\alpha^{a} \frac{1}{r^{\Delta_{1}}}+\beta^{a} \frac{1}{r^{\Delta_{2}}}+\ldots,
$$

where $\Delta_{1,2}$ are the dimensions of the sources and vacuum expectation values (VEV) of the global SU(2) triplet of operators $\mathcal{O}^{a}$ which are dual to the scalar triplet $\phi^{a}$. For our choice of mass, see eq. (2.3), the dimensions are

$$
\Delta_{1}=1, \quad \Delta_{2}=2
$$

and both the $\alpha^{a}$ and the $\beta^{a}$ modes are normalisable. For this reason, we can choose among 
different possible boundary interpretations of the source and VEV: ${ }^{1}$

- the Dirichlet quantisation, where $\alpha^{a}$ corresponds to the source and $\beta^{a}$ to the VEV

$$
J_{D}^{a}=\alpha^{a}, \quad\left\langle\mathcal{O}_{2}^{a}\right\rangle=\beta^{a} .
$$

- the Neumann quantisation, where $-\beta^{a}$ corresponds to the source and $\alpha^{a}$ to the VEV

$$
J_{N}^{a}=-\beta^{a}, \quad\left\langle\mathcal{O}_{1}^{a}\right\rangle=\alpha^{a} .
$$

- the multitrace deformation [54-58], where $\left\langle\mathcal{O}_{1}^{a}\right\rangle=\alpha^{a}$ and the boundary dual is deformed by the action term

$$
S_{\mathcal{F}}=\int d^{3} x \sqrt{-h}\left[J_{\mathcal{F}}^{a} \alpha^{a}+\mathcal{F}\left(\alpha^{a}\right)\right], \quad J_{\mathcal{F}}^{a}=-\beta^{a}-\frac{\partial \mathcal{F}}{\partial \alpha^{a}},
$$

where $\mathcal{F}$ is an arbitrary function. Imposing $J_{\mathcal{F}}^{a}=0$, in order to consider an isolated system, we find the boundary condition

$$
\beta^{a}=-\frac{\partial \mathcal{F}}{\partial \alpha^{a}}
$$

If we use either Dirichlet or Neumann quantisation, there is no non-trivial monopole solution with zero boundary scalar sources. Multitrace deformations, instead, allow finding a monopole solution with a zero boundary source (which satisfies eq. (2.14) for an opportune $\mathcal{F})$, thus in a situation compatible with spontaneous symmetry breaking.

\subsection{Monopole solution in the probe limit}

Let us first consider the zero backreaction limit $G \rightarrow 0$. The monopole solution can be built by a generalisation of 't Hooft-Polyakov ansatz in global $\mathrm{AdS}_{4}$ (see e.g. [41, 42, 51]):

$$
\phi^{a}=\frac{1}{L} H(r) n^{a}, \quad A_{l}^{a}=F(r) r \epsilon^{a i k} n^{k} \partial_{l} n^{i},
$$

where $x^{l}=(r, \theta, \varphi)$ and $n^{k}$ is the unit vector on the sphere $S^{2}$

$$
n^{k}=(\sin \theta \cos \varphi, \sin \theta \sin \varphi, \cos \theta) .
$$

The resulting equations of motion are shown in appendix A.1. The regularity of the solution at small $r$ requires that both $H(r)$ and $F(r)$ approach zero linearly in $r$. On the other hand, at $r \rightarrow \infty$, the choice of boundary conditions depends on the physics we want to describe on the boundary. Such a choice is determined in terms of the coefficients $\left(\alpha_{H}, \beta_{H}, \alpha_{F}, \beta_{F}\right)$ specified in the expansion of the scalar and gauge fields nearby the boundary:

$$
H(r)=\frac{\alpha_{H}}{r}+\frac{\beta_{H}}{r^{2}}+\ldots, \quad F(r)=\frac{\alpha_{F}}{r}+\frac{\beta_{F}}{r^{2}}+\ldots
$$

We choose $\alpha_{F}=0$ in order to describe a theory with spontaneous breaking of the $\mathrm{SU}(2)$ global symmetry, as in [51]. Instead, in order to get a background which is different from

\footnotetext{
${ }^{1}$ The subscript in the operator $\mathcal{O}^{a}$ refers to its dimension.
} 

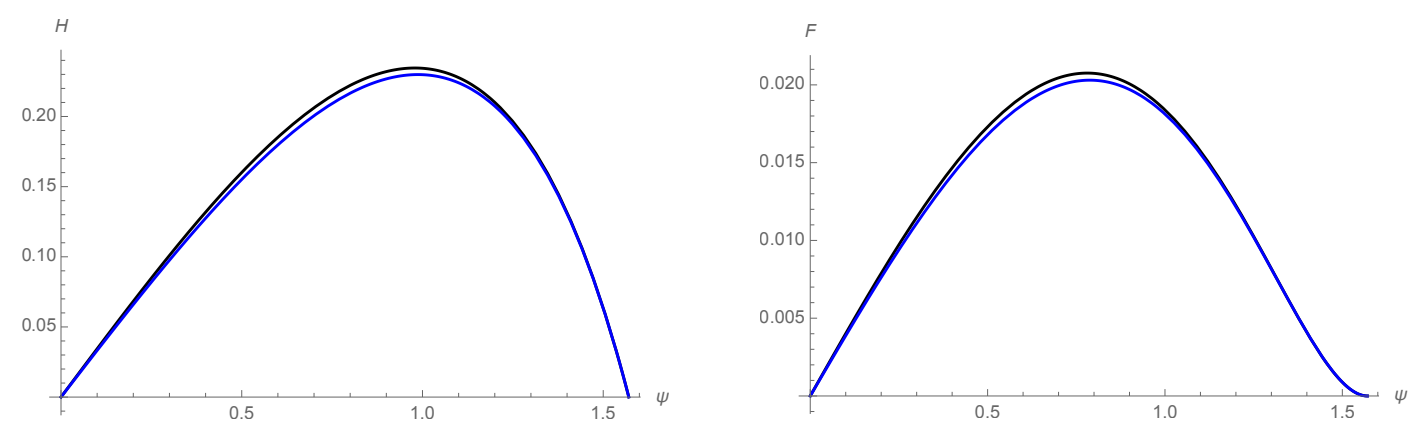

Figure 1: Numerical solutions for $H(\psi)$ and $F(\psi)$ are shown in black (the values $e=1$, $\alpha_{H}=1$ have been used). As a comparison, the analytical approximations (2.18) are shown in blue.

empty AdS, we have to look for solutions where $\alpha_{H}$ and $\beta_{H}$ are generically non-vanishing. Once that $\alpha_{H}$ is fixed, $\beta_{H}$ is determined by the requirement that the solution is smooth. In appendix $\mathrm{B}$ we compute the monopole magnetic flux, which is independent of the boundary conditions expressed in eq. (2.17).

An analytical solution to eqs. (A.1) can be found at the leading order in the gauge coupling $e$ :

$$
\begin{aligned}
& H(r)=\frac{\alpha_{H}}{r}\left[1-\frac{\tan ^{-1} r}{r}\right], \\
& F(r)=\frac{e \alpha_{H}^{2}}{16 r^{3}}\left[\pi^{2} r^{2}-4\left(r^{2}+1\right)\left(\tan ^{-1} r\right)^{2}-\left(\pi^{2}-4\right) r \tan ^{-1} r\right] .
\end{aligned}
$$

Such a solution entails the following coefficients

$$
\beta_{H}=-\frac{\pi}{2} \alpha_{H}, \quad \beta_{F}=e \alpha_{H}^{2} \frac{12 \pi-\pi^{3}}{32} .
$$

The solution (2.18) is also valid for generic $e$, provided that $\alpha_{H}$ is sufficiently small.

At higher order in $e$, eqs. (A.1) can be solved numerically. To this purpose, it is convenient to use the compact variable $\psi$ defined by

$$
r=\tan \psi .
$$

The equations of motion in the variable $\psi$ can be found in appendix A.2. An example of numerical solution is shown in figure 1. A plot of $\left(\alpha_{H}, \beta_{H}\right)$ for various numerical solutions is shown in figure 2 .

\subsection{Monopole backreaction}

We now introduce the monopole backreaction, modelled by the metric

$$
d s^{2}=L^{2}\left(-\left(1+r^{2}\right) h(r) g(r) d \tau^{2}+\frac{h(r)}{g(r)} \frac{d r^{2}}{1+r^{2}}+r^{2}\left(d \theta^{2}+\sin ^{2} \theta d \varphi^{2}\right)\right)
$$




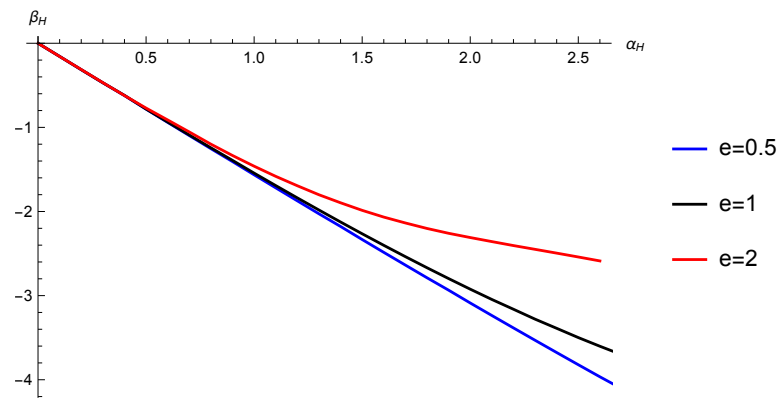

Figure 2: Plot of the relation between the coefficients $\alpha_{H}$ and $\beta_{H}$ for different values of $e$. The relation in eq. (2.19) is perturbatively satisfied.

In order to recover asymptotically global AdS, we impose the following boundary conditions at large $r$

$$
\lim _{r \rightarrow \infty} h=\lim _{r \rightarrow \infty} g=1 .
$$

The full set of equations of motion in this background is given in appendix A.1.

The asymptotic form of the equations of motion fixes the following large $r$ expansions

$$
h(r)=1+\frac{h_{2}}{r^{2}}+\frac{h_{3}}{r^{3}}+O\left(1 / r^{4}\right), \quad g(r)=1+\frac{g_{2}}{r^{2}}+\frac{g_{3}}{r^{3}}+O\left(1 / r^{4}\right),
$$

with

$$
g_{2}=-h_{2}=\frac{2 \pi G \alpha_{H}^{2}}{L^{2}}, \quad h_{3}=-\frac{16 \pi G}{3 L^{2}} \alpha_{H} \beta_{H} .
$$

The unfixed parameter $g_{3}$ can be found by requiring that the solution is smooth.

In order to treat the problem in an analytical way, it is useful to introduce the expansion parameter

$$
\epsilon=\frac{\pi G \alpha_{H}^{2}}{L^{2}}
$$

At the leading order in $e$ and in $\epsilon$, the $H(r)$ and $F(r)$ solutions are still given by eq. (2.18). The leading order backreaction on the metric can be solved analytically too, giving:

$$
h(r)=1+\epsilon h_{\epsilon}+O\left(\epsilon^{2}\right), \quad g(r)=1+\epsilon g_{\epsilon}+O\left(\epsilon^{2}\right),
$$

where

$$
\begin{aligned}
h_{\epsilon}= & \pi^{2}-\frac{4}{r^{2}}-\frac{2}{r^{2}+1}-4 \frac{2\left(r^{2}-1\right) r \tan ^{-1} r+\left(r^{4}+1\right)\left(\tan ^{-1} r\right)^{2}}{r^{4}}, \\
g_{\epsilon}= & \pi^{2}+\frac{1}{r^{2}}-\frac{2 r \tan ^{-1} r+3}{r^{2}}\left(1-\frac{2}{1+r^{2}}\right) \\
& -2\left(\tan ^{-1} r\right) \frac{2\left(r^{4}-1\right) \tan ^{-1} r+r\left(3 r^{2}+4\right)}{r^{4}} .
\end{aligned}
$$

These solutions set

$$
g_{3}=-\frac{10 \pi^{2} G \alpha_{H}^{2}}{3 L^{2}} .
$$



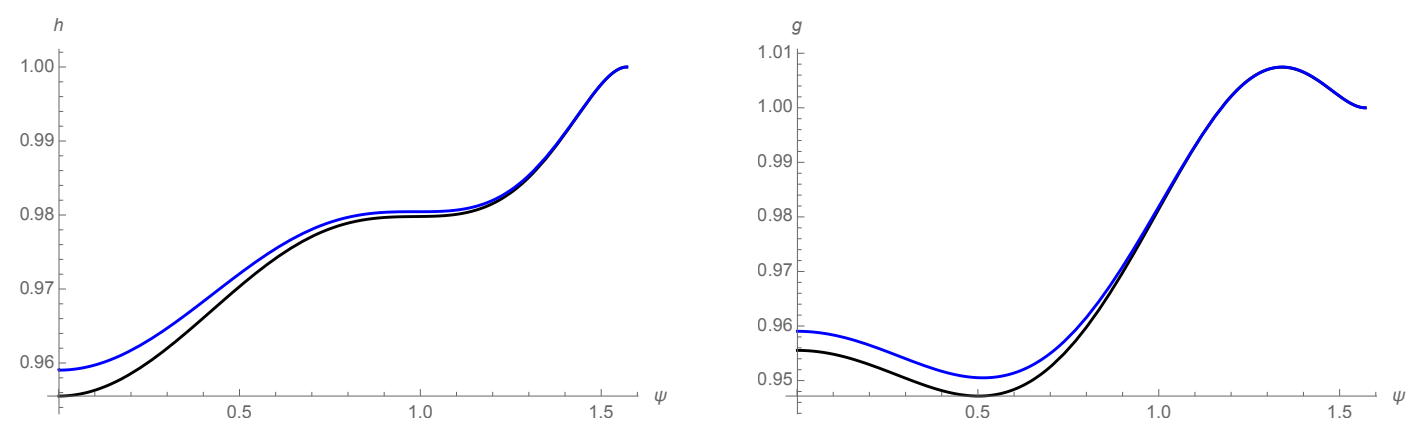

Figure 3: Numerical solutions for the metric functions $h(\psi)$ and $g(\psi)$ are shown in black (the values $e=1, \alpha_{H}=1, L=1$ and $G=0.1$ have been used). As a comparison, the analytical approximations (2.27) are shown in blue.

The profile functions at higher order in $e$ and $\epsilon$ are again accessible by numerically solving the equations of motion. As in the probe limit, it is convenient to introduce the variable $\psi=\tan ^{-1} r$, getting the equations of motion shown in appendix A.2. A comparison between the numerical and the analytical solutions is shown in figure 3 .

\section{A falling monopole in Poincaré patch}

The gravity dual of a local quench in a CFT can be realised by considering a falling particle in AdS [31]. To this purpose, a nice trick was introduced in [52]. The idea is to start from a spherically symmetric geometry in global AdS, and to transform it to a time-dependent Poincaré AdS geometry by performing a change of variables.

The Poincaré $\mathrm{AdS}_{4}$ metric with coordinates $(t, z, x, \varphi)$ is

$$
d s^{2}=L^{2}\left(\frac{d z^{2}-d t^{2}+d x^{2}+x^{2} d \varphi^{2}}{z^{2}}\right) .
$$

The metric in eq. (3.1) and the global AdS metric in eq. (2.8) can be mapped into each other via the coordinate transformations

$$
\begin{aligned}
\sqrt{1+r^{2}} \cos \tau & =\frac{A^{2}+z^{2}+x^{2}-t^{2}}{2 A z}, & \sqrt{1+r^{2}} \sin \tau & =\frac{t}{z}, \\
r \sin \theta & =\frac{x}{z}, & r \cos \theta & =\frac{z^{2}+x^{2}-t^{2}-A^{2}}{2 A z},
\end{aligned}
$$

leaving the angular coordinate $\varphi$ unchanged.

These transformations can be inverted as follows:

$$
\begin{aligned}
& r=\frac{\sqrt{A^{4}+2 A^{2}\left(t^{2}+x^{2}-z^{2}\right)+\left(z^{2}+x^{2}-t^{2}\right)^{2}}}{2 A z}, \\
& \tau=\tan ^{-1}\left(\frac{2 A t}{z^{2}+x^{2}-t^{2}+A^{2}}\right), \\
& \theta=\tan ^{-1}\left(\frac{2 A x}{z^{2}+x^{2}-t^{2}-A^{2}}\right) .
\end{aligned}
$$


The change of variables in eq. (3.3) maps a configuration with a static particle in the center of global AdS to a falling particle in the Poincaré patch, that can be used to model a local quench. We will apply this method to the monopole solution we discussed in section 2 .

The holographic quench is symmetric under time reversal $t \rightarrow-t$ : for $t<0$ the monopole is approaching the boundary, while for $t>0$ it moves in the direction of the bulk interior. Physically, we can think of the initial condition at $t=0$ as the initial outof-equilibrium state, which can be prepared in the dual conformal field theory by some appropriate operator insertion.

The position of the monopole center, corresponding to $r=0$ in global AdS, in the Poincaré patch is time-dependent and follows the curve

$$
x=0, \quad z=\sqrt{t^{2}+A^{2}} .
$$

In the approximation in which the monopole is a pointlike particle, eq. (3.4) can be interpreted as the trajectory of the monopole. From the gravity side, the parameter $A$ can be interpreted as the initial position along the $z$-direction of the free-falling monopole. From the CFT perspective, the parameter $A$ fixes the size of the local quench.

\subsection{Bulk energy density of the falling monopole}

One may be tempted to imagine the monopole as a pointlike particle which is falling along the trajectory in eq. (3.4). To check this intuition, it is natural to consider the bulk energy-momentum tensor (2.7).

Working in the limit of negligible monopole backreaction, we perform the coordinate change in eq. (3.3)

$$
x^{\mu}=(\tau, r, \theta, \varphi) \rightarrow x^{\prime \mu}=(t, z, x, \varphi)
$$

The energy-momentum tensor in Poincaré patch is given by

$$
T_{\alpha \beta}^{\prime}\left(x^{\prime}\right)=\frac{\partial x^{\mu}}{\partial x^{\prime \alpha}} \frac{\partial x^{\nu}}{\partial x^{\prime \beta}} T_{\mu \nu}(x) .
$$

To properly normalise the energy-momentum tensor, we introduce the vierbein $e_{m}^{\mu}$ such that

$$
T_{m n}^{\prime}\left(x^{\prime}\right)=e_{m}^{\mu} e_{n}^{\nu} T_{\mu \nu}^{\prime}\left(x^{\prime}\right), \quad e_{m}^{\mu} e_{n}^{\nu} g_{\mu \nu}^{\prime}=\eta_{m n}
$$

where $g_{\mu \nu}^{\prime}$ and $\eta_{m n}$ are the Poincaré AdS and the Minkowski metric tensors, respectively. In particular, we choose ${ }^{2}$

$$
e_{0}^{\mu}=\left(\frac{z}{L}, 0,0,0\right), \quad e_{1}^{\mu}=\left(0, \frac{z}{L}, 0,0\right), \quad e_{2}^{\mu}=\left(0,0, \frac{z}{L}, 0\right) .
$$

The energy density as measured in such an orthonormal frame is

$$
\rho=T^{\prime 00}=\frac{z^{2}}{L^{2}} T_{t t}^{\prime}
$$

\footnotetext{
${ }^{2}$ In this section, the Minkowski indices $m, n$ take the values $0,1,2,3$, while the curved spacetime indices are $t, z, x, \varphi$.
} 

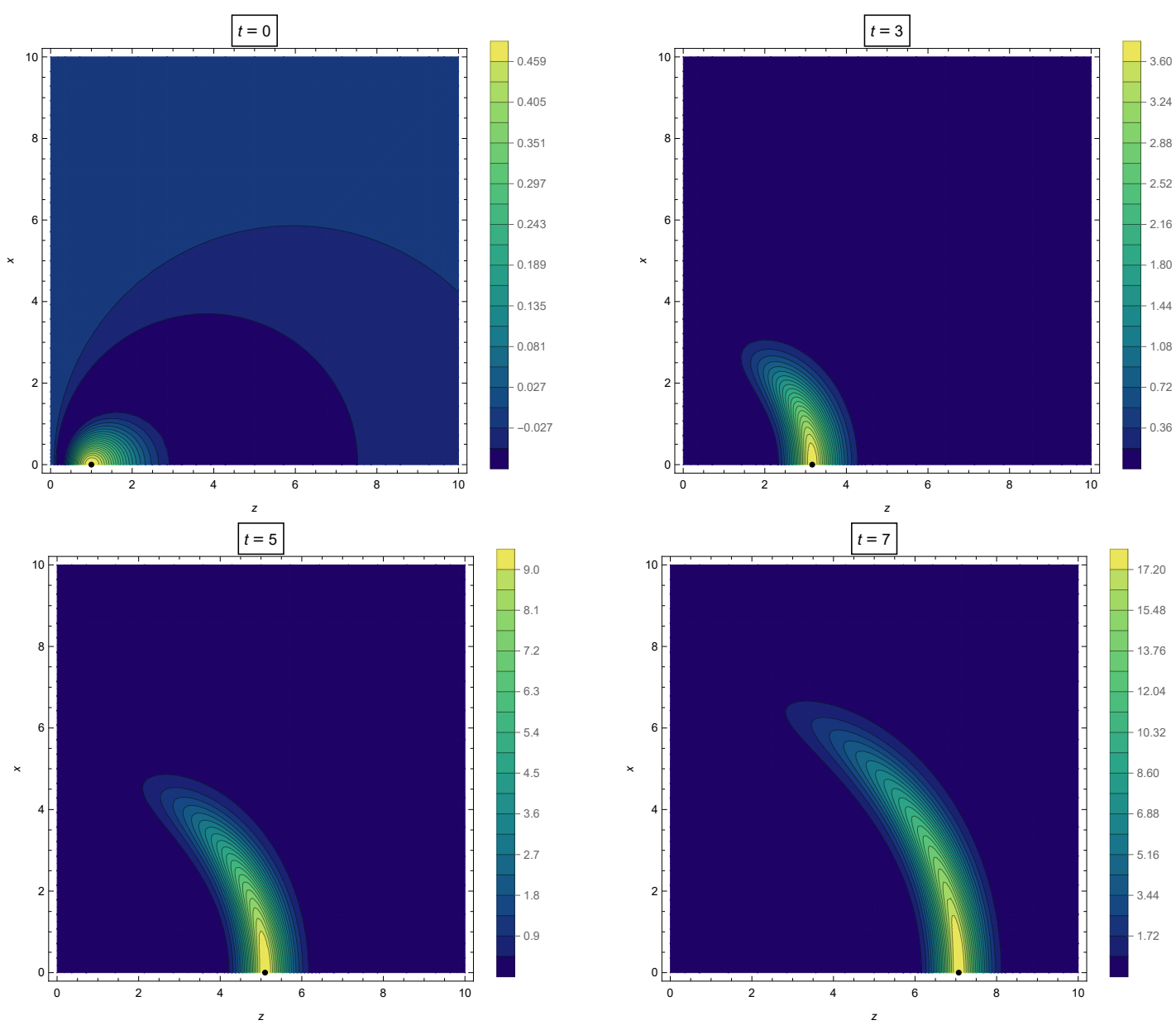

Figure 4: Contour lines of constant energy density for fixed time. The monopole center is represented by the black spot. The numerical values $A=1$ and $L=1$ have been chosen.

and the components of the Poynting vector $\vec{s}=\left(s_{z}, s_{x}, s_{\varphi}\right)$ are

$$
s_{z}=T^{\prime 01}=-\frac{z^{2}}{L^{2}} T_{t z}^{\prime}, \quad s_{x}=T^{\prime 02}=-\frac{z^{2}}{L^{2}} T_{t x}^{\prime}, \quad s_{\varphi}=0 .
$$

In figure 4 and 5 we show the numerical results for the energy density and the energy flux into the bulk at fixed time.

The pictures clearly illustrate how the energy density, initially localised near the AdS boundary, spreads into the bulk. The energy distribution resembles that of a pointlike particle only at early times, while at late times the energy is spread along a spherical wavefront. At $t=0$ all the components of the Poynting vector vanish, implying that there is no energy flux at initial time.

\section{Expectation values of local operators}

To understand the physical interpretation on the boundary CFT, it is useful to study the expectation values of some important local operators. In particular, in this section we 


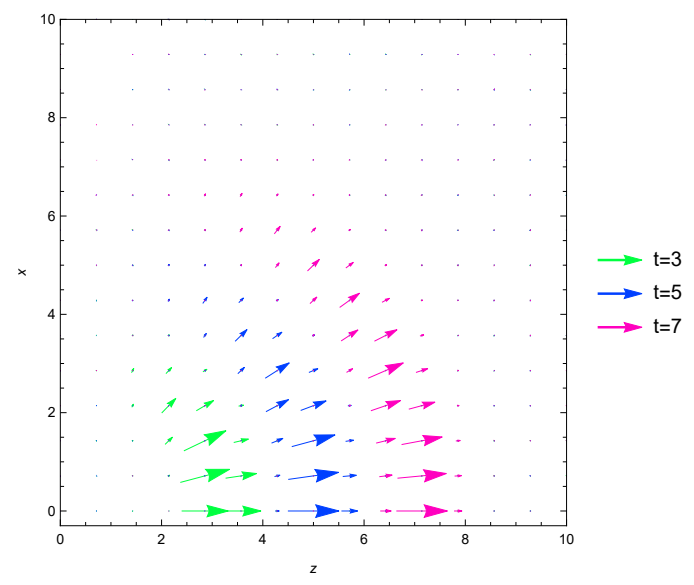

Figure 5: Direction of the bulk Poynting vector for fixed time. The numerical values $A=1$ and $L=1$ have been chosen.

will focus on expectation values of scalar operators, global SU(2) currents and energymomentum tensor. The details depend on the boundary conditions chosen for the scalar field $\phi^{a}$. In general, the boundary energy-momentum tensor $T_{m n}$ is not conserved because the external sources perform work on the system. With a particular choice of multitrace deformation, see eq. (4.14), the system is isolated and $T_{m n}$ is conserved.

\subsection{Boundary conditions for the scalar}

In the AdS/CFT correspondence, the asymptotic behaviour of the bulk fields is dual to the source and expectation values of operators in the CFT. For this reason, we will focus on asymptotics of the scalar field $\phi^{a}$ nearby the boundary. In global AdS, the direction $n^{a}$ of $\phi^{a}$ in the internal SU(2) space is given by eq. (2.16). In Poincaré patch, by performing the coordinate transformation in eq. (3.3) we find that, nearby the boundary at $z=0$,

$$
n^{a}=\frac{1}{\omega^{1 / 2}}\left(-2 A x \cos (\varphi),-2 A x \sin (\varphi), A^{2}+t^{2}-x^{2}\right)+O\left(z^{2}\right)
$$

where, for convenience, we introduce the quantity $\omega(x, t)$ that appears in many subsequent expressions

$$
\omega(x, t)=A^{4}+2 A^{2}\left(t^{2}+x^{2}\right)+\left(t^{2}-x^{2}\right)^{2} .
$$

The core of the quench can be thought of as localised at

$$
x=\sqrt{t^{2}+A^{2}}
$$

which, at large $t$, coincides with good approximation with the lightcone of the origin $x=t$. For the value in eq. (4.3), the adjoint scalar field points in the direction $n=n^{a} \sigma^{a}$ given by

$$
n=-\left(\sigma_{1} \cos \varphi+\sigma_{2} \sin \varphi\right) .
$$

The scalar points along the $\sigma_{3}$ direction inside the lightcone, and along the $-\sigma_{3}$ outside the lightcone, see figure 6 . As we will see later, at large $t$, the absolute value of the scalar field is peaked on $x$ given by eq. (4.3), and is almost zero both inside and outside the lightcone. 


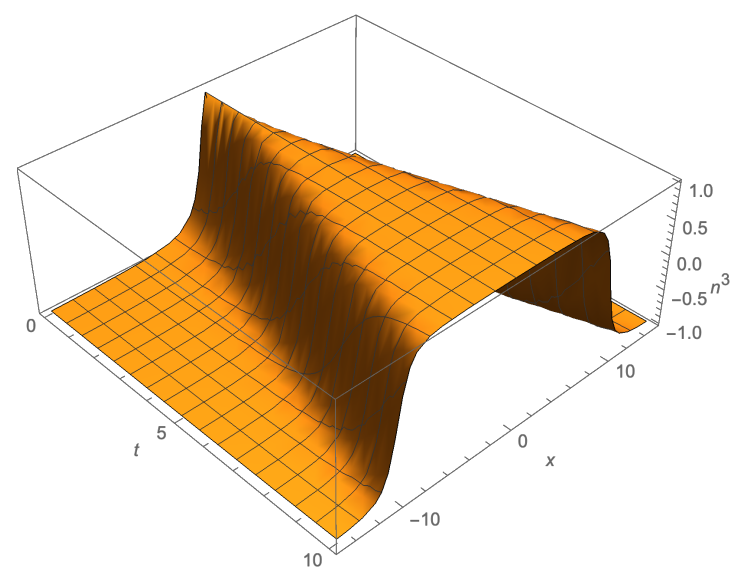

Figure 6: Value of $n^{3}$ as a function of $(t, x)$ for $A=1$. Negative values of the radial cylindrical coordinate $x$ correspond to $\varphi \rightarrow-\varphi$.

The configuration at $t=0$ resembles a baby skyrmion, with a field pointing along $\sigma_{3}$ in the core and along $-\sigma_{3}$ far away (actually, it is not a skyrmion because the VEV tends to zero at infinity). As time increases, this configuration expands along the lightcone. At large time we end up with two regions of vacuum (inside and outside the lightcone) separated by an expanding shell of energy.

In order to extract the sources and the expectation values of the local operator triggering the quench, it is useful to expand the change of variables in eq. (3.3) nearby the boundary. The global AdS radial coordinate reads

$$
r=\frac{a}{z}+O(z), \quad a=\frac{\omega^{1 / 2}}{2 A} .
$$

By means of eq. (4.5), we obtain the boundary expansion of $H(r)$

$$
H=\frac{\alpha_{H}}{r}+\frac{\beta_{H}}{r^{2}}+O\left(r^{-3}\right)=\tilde{\alpha}_{H} z+\tilde{\beta}_{H} z^{2}+O\left(z^{3}\right),
$$

where

$$
\tilde{\alpha}_{H}=\frac{\alpha_{H}}{a}=\alpha_{H} \frac{2 A}{\omega^{1 / 2}}, \quad \tilde{\beta}_{H}=\frac{\beta_{H}}{a^{2}}=\beta_{H} \frac{4 A^{2}}{\omega} .
$$

A plot of $\tilde{\alpha}_{H}$ and $\tilde{\beta}_{H}$ is shown in figure 7 . It is interesting to note that

$$
\frac{\tilde{\beta}_{H}}{\tilde{\alpha}_{H}^{2}}=\frac{\beta_{H}}{\alpha_{H}^{2}}=\kappa,
$$

where $\kappa$ is a constant. In the limit of small backreaction, from eq. (2.19) we find

$$
\kappa=-\frac{\pi}{2 \alpha_{H}} .
$$

Combining eqs. (4.1) and (4.6), the expansion of $\phi^{a}$ nearby the Poincaré patch boundary is

$$
\phi^{a}=\frac{H(z)}{L} n^{a}=\frac{1}{L}\left(\tilde{\alpha}^{a} z+\tilde{\beta}^{a} z^{2}+O\left(z^{3}\right)\right), \quad \tilde{\alpha}^{a}=n^{a} \tilde{\alpha}_{H}, \quad \tilde{\beta}^{a}=n^{a} \tilde{\beta}_{H} .
$$



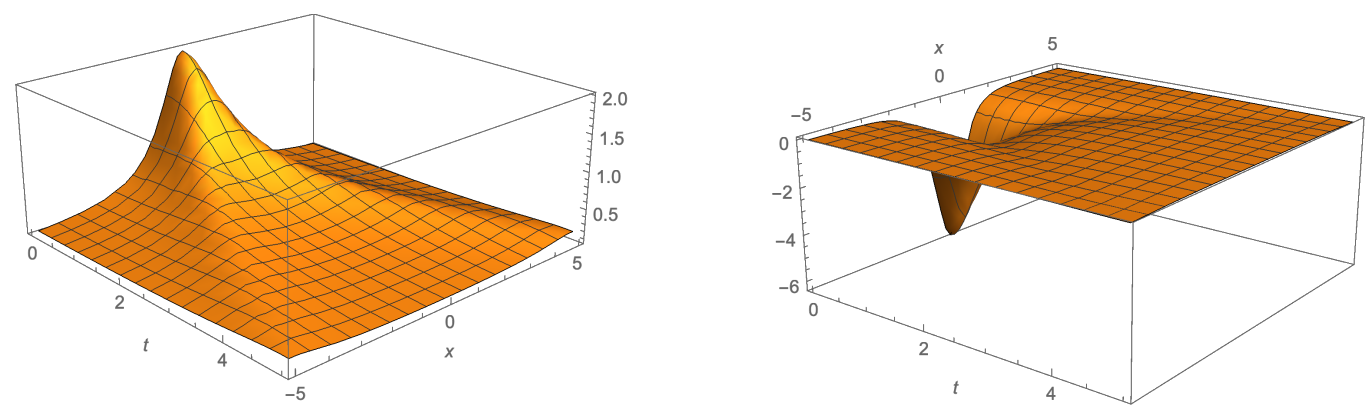

Figure 7: The quantities $\tilde{\alpha}_{H}$ (left) and $\tilde{\beta}_{H}$ (right) as a function of $(t, x)$. Here we set $A=1, \alpha_{H}=1$ and we use the relations in eq. (2.19), valid for small backreaction.

As for the global AdS case, we can consider several quantisations for the scalar field $\phi^{a}$ :

- the Dirichlet condition, where $\tilde{\alpha}_{H}$ corresponds to the source and $\tilde{\beta}_{H}$ to the VEV

$$
J_{D}^{a}=\tilde{\alpha}^{a}, \quad\left\langle\mathcal{O}_{2}^{a}\right\rangle=\tilde{\beta}^{a} .
$$

- the Neumann condition, where $-\tilde{\beta}_{H}$ corresponds to the source and $\tilde{\alpha}_{H}$ to the VEV

$$
J_{N}^{a}=-\tilde{\beta}^{a}, \quad\left\langle\mathcal{O}_{1}^{a}\right\rangle=\tilde{\alpha}^{a} .
$$

- the multitrace deformation, where the boundary dual is deformed by the action term

$$
S_{\mathcal{F}}=\int d^{3} x \sqrt{-h}\left[J_{\mathcal{F}}^{a} \tilde{\alpha}^{a}+\mathcal{F}\left(\tilde{\alpha}^{a}\right)\right], \quad J_{\mathcal{F}}^{a}=-\tilde{\beta}^{a}-\frac{\partial \mathcal{F}}{\partial \tilde{\alpha}^{a}},
$$

and $\left\langle\mathcal{O}_{1}^{a}\right\rangle=\tilde{\alpha}^{a}$.

All these boundary conditions correspond in general to a monopole in presence of external time-dependent sources. Among such possible choices of boundary conditions, it is interesting to consider the multitrace deformation with

$$
\mathcal{F}_{\kappa}\left(\tilde{\alpha}^{a}\right)=-\frac{\kappa}{3}\left(\tilde{\alpha}^{a} \tilde{\alpha}^{a}\right)^{3 / 2}=-\frac{\kappa}{3} \tilde{\alpha}_{H}^{3} .
$$

In this case, the monopole is a solution with a vanishing source, because it satisfies

$$
\tilde{\beta}^{a}=-\frac{\partial \mathcal{F}}{\partial \tilde{\alpha}^{a}},
$$

as can be checked from eq. (4.8).

\subsection{The boundary global currents}

Our monopole ansatz in global AdS is given by eq. (2.15), with boundary conditions in eq. (2.17) and with $\alpha_{F}=0$. As a consequence, we deduce that in Poincaré patch the gauge field $A_{\mu}^{a}$ vanishes at the boundary $z=0$. In other words, if the sources for the global symmetries are set to zero in global AdS, they also vanish after the change of coordinates leading to the Poincaré patch. 


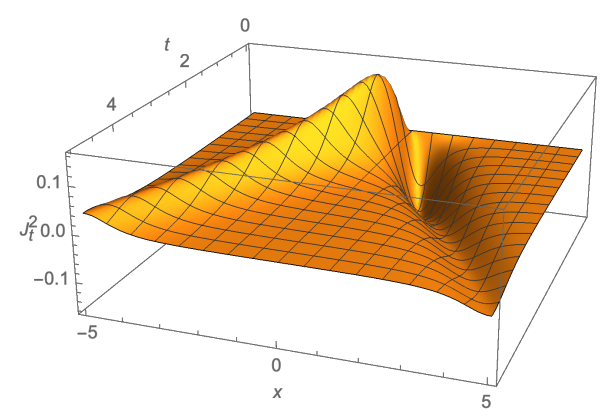

Figure 8: Charge density of the second component of isospin $J_{t}^{2}$ as a function of $(t, x)$ for $\varphi=0$ (positive $x$ ) and $\varphi=\pi$ (negative $x$ ). We set $A=1, \alpha_{H}=1$ and $e=1$ and we use the relations in eq. (2.19), valid for small backreaction. The peak and the pit correspond to positive and negative sign global charges, which are taken apart from each other by the quench.

From the order $z$ terms in the boundary expansion of $A_{\mu}^{a}$ we can extract the expectation value of the three currents $J_{l}^{a}$

$$
\begin{aligned}
& \left\langle J_{l}^{1}\right\rangle=\frac{8 A^{2} \beta_{F}}{\omega^{3 / 2}}\left(t x \sin (\varphi),-\frac{1}{2} \sin (\varphi)\left(A^{2}+t^{2}+x^{2}\right),-\frac{1}{2} x \cos (\varphi)\left(A^{2}+t^{2}-x^{2}\right)\right), \\
& \left\langle J_{l}^{2}\right\rangle=\frac{8 A^{2} \beta_{F}}{\omega^{3 / 2}}\left(-t x \cos (\varphi), \frac{1}{2} \cos (\varphi)\left(A^{2}+t^{2}+x^{2}\right),-\frac{1}{2} x \sin (\varphi)\left(A^{2}+t^{2}-x^{2}\right)\right), \\
& \left\langle J_{l}^{3}\right\rangle=\frac{8 A^{2} \beta_{F}}{\omega^{3 / 2}}\left(0,0,-A x^{2}\right),
\end{aligned}
$$

where $x^{l}=(t, x, \varphi)$ are the boundary spacetime coordinates, and $a=1,2,3$ is the $\mathrm{SU}(2)$ global index. Plots of the charge density $J_{t}^{2}$ is shown in figure 8 .

It is interesting to compare the direction in the $\mathrm{SU}(2)$ space of the current expectation value with the direction of the scalar expectation value $n^{a}$. We find that the expectation value of the global current is always orthogonal to the direction of the scalar expectation value in the isospin space

$$
\left\langle J_{m}^{a}\right\rangle n^{a}=0 .
$$

So the quench breaks all the three global symmetry group generators. This is true just on top of the "lightcone" in eq. (4.3); inside and outside this surface both the scalar and the current expectation values tend to zero and the $\mathrm{SU}(2)$ global symmetry of the boundary theory is unbroken.

\subsection{The boundary energy-momentum tensor}

For illustrative purposes, we will compare the result with the one obtained for a quench modelled by a falling black hole, studied in [31]. In this case the metric is

$$
d s^{2}=L^{2}\left(-\left(1+r^{2}-\frac{M}{r}\right) d \tau^{2}+\frac{d r^{2}}{1+r^{2}-\frac{M}{r}}+r^{2}\left(d \theta^{2}+\sin ^{2} \theta d \varphi^{2}\right)\right)
$$


where $M$ is a dimensionless parameter proportional to the black hole mass

$$
m_{B H}=\frac{1}{2} \frac{M L}{G} .
$$

In order to find the quench background, we apply the change of variables in eq. (3.3). Then, to extract the energy-momentum tensor with the method in [59], it is convenient to pass to Fefferman-Graham (FG) coordinates. Details of the calculation are in appendix C.1, where expressions for all the components of the energy-momentum tensor can also be found, see eq. (C.7). In particular, the energy density is

$$
T_{t t}^{(B H)}=\frac{A^{3} L^{2} M}{\pi G} \frac{\omega+6 t^{2} x^{2}}{\omega^{5 / 2}} .
$$

The energy-momentum tensor is conserved and traceless, and the total energy is

$$
\mathcal{E}^{(B H)}=\frac{L}{A} m_{B H}
$$

In the falling monopole case a more accurate discussion is needed, since the boundary energy-momentum tensor depends on the details of the boundary conditions of bulk fields. We first focus on Dirichlet boundary conditions [60]. Starting from the backreacted metric in eq. (2.21), we apply the change of variables in eq. (3.3). The intermediate metric expression is a bit cumbersome, so in appendix C.2 we just specify the coordinates expansion that puts it in the FG form. All the non-vanishing components of the energy-momentum tensor $T_{m n}^{(D)}$ obtained from such a metric are given in eq. (C.10). The energy density is:

$$
\begin{aligned}
T_{t t}^{(D)} & =\frac{A^{3}}{3 \pi G \omega^{5 / 2}}\left[48 \pi G \alpha_{H} \beta_{H} x^{2} t^{2}+\left(8 \pi G \alpha_{H} \beta_{H}-3 g_{3} L^{2}\right)\left(\omega+6 t^{2} x^{2}\right)\right] \\
& =2 \pi \alpha_{H}^{2} A^{3} \frac{\omega+2 t^{2} x^{2}}{\omega^{5 / 2}},
\end{aligned}
$$

where in the second line we have inserted the analytic approximations for small backreaction in eqs. (2.19) and (2.28). In this limit, the total energy is

$$
\mathcal{E}^{(D)}=\frac{\pi^{2} \alpha_{H}^{2}}{A}\left(1-\frac{2}{3} \frac{t^{2}}{A^{2}+t^{2}}\right) .
$$

The energy is a decreasing function of time, meaning that the Dirichlet boundary conditions absorb energy from the bulk. The non-conservation of energy motivates the investigation of a different quantisation.

A changing of the quantisation conditions causes a shift of $T_{m n}^{(D)}$ by finite parts (see e.g. [58]). Here we specialise to a class of multitrace deformations that do not break the $\mathrm{SU}(2)$ global symmetry. Assuming that

$$
\tilde{\alpha}^{a}=n^{a} \tilde{\alpha}_{H}, \quad \tilde{\beta}^{a}=n^{a} \tilde{\beta}_{H},
$$

which is true for the monopole, we can write the source as

$$
J_{\mathcal{F}}^{a}=n^{a} J_{\mathcal{F}}
$$


As a function $\mathcal{F}$ parameterising the multitrace deformation we choose

$$
\mathcal{F}\left(\tilde{\alpha}^{a}\right)=\mathcal{F}\left(\tilde{\alpha}^{a} \tilde{\alpha}^{a}\right)=\mathcal{F}\left(\alpha_{H}\right) .
$$

The current can be written in terms of $\tilde{\alpha}_{H}, \tilde{\beta}_{H}$ as follows

$$
J_{\mathcal{F}}=-\tilde{\beta}_{H}-\mathcal{F}^{\prime}\left(\tilde{\alpha}_{H}\right) .
$$

The energy-momentum tensor, (see appendix C.3 for further details) is

$$
T_{i j}^{(\mathcal{F})}=T_{i j}^{(D)}+\eta_{i j}\left[\mathcal{F}\left(\tilde{\alpha}_{H}\right)-\tilde{\alpha}_{H} \tilde{\beta}_{H}-\mathcal{F}^{\prime}\left(\tilde{\alpha}_{H}\right) \tilde{\alpha}_{H}\right]
$$

Note that this result also applies to the Neumann conditions, that can be seen as a multitrace deformation with $\mathcal{F}=0$. If we instead specialise to $\mathcal{F}=\mathcal{F}_{\kappa}$, see eq. (4.14), the external source is zero and the energy-momentum tensor is conserved. Moreover, an explicit computation reveals that the energy-momentum tensor has the same functional form as the one for the falling $\mathrm{BH}$ :

$$
T_{i j}^{(\kappa)}=\frac{m_{M}}{m_{B H}} T_{i j}^{(B H)}, \quad m_{M}=\frac{16 \pi G \alpha_{H} \beta_{H}-3 L^{2} g_{3}}{6 L G},
$$

where $m_{M}$ is the monopole mass. Using the analytical values in eqs. (2.19) and (2.28), we find

$$
m_{M}=\frac{\pi^{2}}{3} \frac{\alpha_{H}^{2}}{L}=\frac{\pi}{3} \frac{L}{G} \epsilon
$$

The total energy is

$$
\mathcal{E}^{(\kappa)}=\frac{L}{A} m_{M}
$$

As apparent from eq. (4.29), the energy-momentum tensor is not a probe enough precise to distinguish between a falling monopole or a falling black hole in the bulk. In the next section, we will see that the entanglement entropy of these two falling objects behaves instead in a radically different way.

\section{Holographic entanglement entropy}

In this section we will study the effect of the leading order backreaction on holographic entanglement entropy. It is useful to use $\epsilon$ as defined in eq. (2.25) as an expansion parameter. We will find that the corrections to the entropy due to the classical backreaction are of order $\alpha_{H}^{2}$, where

$$
\alpha_{H}^{2}=\epsilon \frac{L^{2}}{G} \frac{1}{\pi} .
$$

In the limit of extremely small $\epsilon$, bulk quantum corrections [61] can be of the same order of magnitude as the ones due to classical backreaction (see for example [36]). Here we will focus on a regime where the quantum corrections are negligible. We first fix an $\epsilon$ sufficiently small in order to justify the calculation of the backreaction at the leading order. Then, we choose

$$
\frac{L^{2}}{G} \gg \frac{1}{\epsilon}
$$


in such a way that the classical bulk contributions dominate over the quantum ones, which are of order $\left(L^{2} / G\right)^{0}$. This corresponds to a large monopole mass, i.e. $m_{M} \gg 1 / L$, see eq. (4.30), which means that the dual local operator triggering the quench has a large operator dimension, as in [30]. In order to trust our analytical approximation for a sufficiently large $\alpha_{H}^{2}$, we need to choose the gauge coupling $e$ sufficiently small, see the discussion below eq. (2.19).

In the asymptotically global AdS case, the metric at the leading order in $\epsilon$ is

$$
d s^{2}=L^{2}\left(-\left(1+r^{2}\right)\left[1+\epsilon\left(h_{\epsilon}+g_{\epsilon}\right)\right] d \tau^{2}+\left[1+\epsilon\left(h_{\epsilon}-g_{\epsilon}\right)\right] \frac{d r^{2}}{1+r^{2}}+r^{2}\left(d \theta^{2}+\sin ^{2} \theta d \varphi^{2}\right)\right)
$$

where $h_{\epsilon}$ and $g_{\epsilon}$ are defined in eq. (2.26). We will be interested in the evolution of entanglement entropy for the quench in Poincaré patch, so we apply the change of variables in eq. (3.3), obtaining a time-dependent background.

The metric tensor can be written as follows

$$
g_{\mu \nu}=g_{\mu \nu}^{(0)}+\epsilon g_{\mu \nu}^{(1)}+O\left(\epsilon^{2}\right), \quad \epsilon=\frac{\pi G \alpha_{H}^{2}}{L^{2}} .
$$

Given a codimension-two surface $x^{\mu}\left(y^{\alpha}\right)$ parameterised with coordinates $y^{\alpha}=\left(y^{1}, y^{2}\right)$, the induced metric is

$$
G_{\alpha \beta}=\frac{\partial x^{\mu}}{\partial y^{\alpha}} \frac{\partial x^{\nu}}{\partial y^{\beta}} g_{\mu \nu}
$$

Such an induced metric can also be expanded as a power series in $\epsilon$

$$
G_{\alpha \beta}=G_{\alpha \beta}^{(0)}+\epsilon G_{\alpha \beta}^{(1)}+O\left(\epsilon^{2}\right), \quad G_{\alpha \beta}^{(k)}=\frac{\partial x^{\mu}}{\partial y^{\alpha}} \frac{\partial x^{\nu}}{\partial y^{\beta}} g_{\mu \nu}^{(k)}, \quad k=0,1 .
$$

We can compute the change of area of the Ryu-Takayanagi (RT) surface at the leading order in $\epsilon$, as in [31]. To this purpose, we can expand the determinant of the metric in the area functional. The first order term of this expansion is

$$
\Delta \mathcal{A}=\frac{\epsilon}{2} \int d^{2} y \sqrt{G^{(0)}} \operatorname{Tr}\left[G^{(1)}\left(G^{(0)}\right)^{-1}\right] .
$$

It is important to note that, at first order, it is enough to work with the unperturbed RT surface $x^{\mu}\left(y^{\alpha}\right)$, which simplifies the computation a lot. The difference in entropy between the excited state and the vacuum at the leading order is proportional to eq. (5.7)

$$
\Delta S=\frac{\Delta \mathcal{A}}{4 G} .
$$

We will apply this procedure to various examples of subregions.

\subsection{Disk centered at the origin}

We take as a boundary subregion a disk of radius $l$ centered at $x=0$ and lying at constant time $t$. The RT surface in unperturbed Poincaré patch of $\mathrm{AdS}_{4}$ is the half sphere

$$
z=\sqrt{l^{2}-x^{2}} .
$$



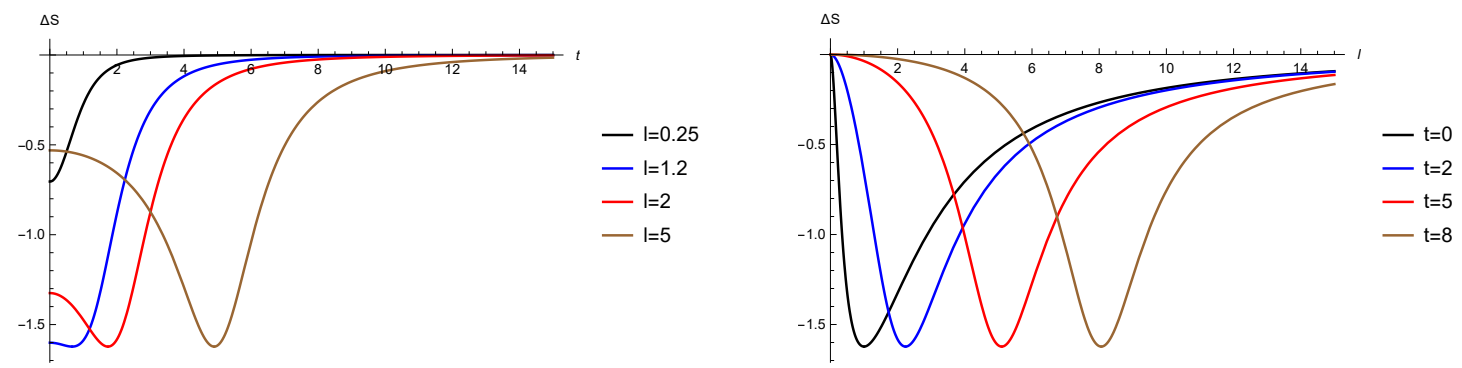

Figure 9: Left: time dependence of $\Delta S$ for a spherical subregion with fixed radius $l$ centered at the origin of the quench. Right: dependence of $\Delta S$ at fixed $t$ as a function of $l$. The numerical values $\alpha_{H}=1, A=1$ are used.

From eqs. (5.7) and (5.3) we obtain

$$
\Delta S(l, t)=\frac{\pi^{2} \alpha_{H}^{2}}{4} \frac{1}{l} \int_{0}^{l} \frac{\left(h_{\epsilon}-g_{\epsilon}\right) x^{3}}{\left(l^{2}-x^{2}\right)^{3 / 2}} \frac{\omega(l, t)}{\left(A^{2}-l^{2}+t^{2}\right)^{2}+4 A^{2} x^{2}} d x,
$$

where $\omega$ is defined in eq. (4.2). The functions $h_{\varepsilon}$ and $g_{\varepsilon}$ depend on the variable $r$, which on top of the RT surface reads

$$
r=\frac{\sqrt{\left(A^{2}-l^{2}+t^{2}\right)^{2}+4 A^{2} x^{2}}}{2 A \sqrt{l^{2}-x^{2}}} .
$$

For the entropy, the $A$ dependence can be completely reabsorbed by the following rescaling of the quantities $l, x$ and $t$

$$
l \rightarrow \frac{l}{A}, \quad x \rightarrow \frac{x}{A}, \quad t \rightarrow \frac{t}{A} .
$$

For this reason, the numerical analysis has been performed for $A=1$ without loss of generality. Numerical results are shown in figure 9. We find that $\Delta S$ is always negative, meaning that the perturbed entanglement entropy is always smaller than the vacuum value. We can think of the quench as a region of spacetime where a condensate (which breaks a global symmetry on the boundary, as in holographic superconductors [62]) is localised. A lower entropy fits with the intuition that some degrees of freedom have condensed [53] and so there should be fewer of them compared to the vacuum (which has zero scalar expectation value).

Analytical results can be found in some regimes. Nearby the boundary $r \rightarrow+\infty$, we can use the expansion

$$
h_{\epsilon}=-\frac{2}{r^{2}}+\ldots, \quad g_{\epsilon}=\frac{2}{r^{2}}+\ldots .
$$

Since the minimal $r$ on the RT surface is given by eq. (5.11) with $x=0$, this expansion will be valid in the whole integration region in eq. (5.10) in the regime

$$
\left|A^{2}+t^{2}-l^{2}\right| \gg 2 l A
$$


Equation (5.10) can then be evaluated explicitly

$$
\Delta S=-\pi^{2} \alpha_{H}^{2}\left[\left(\frac{A l}{\sqrt{\omega(l, t)}}+\frac{\sqrt{\omega(l, t)}}{4 A l}\right) \tanh ^{-1}\left(\frac{2 A l}{\sqrt{\omega(l, t)}}\right)-\frac{1}{2}\right] .
$$

We can specialise the approximation in eq. (5.14) to the following situations:

- small $l \ll A$

$$
\Delta S=-\frac{8}{3} \pi^{2} \alpha_{H}^{2} \frac{A^{2} l^{2}}{\left(A^{2}+t^{2}\right)^{2}}
$$

- large $t \gg A$ and $t \gg l$

$$
\Delta S=-\frac{8}{3} \pi^{2} \alpha_{H}^{2} \frac{l^{2} A^{2}}{t^{4}}
$$

- $t=0$ and $l \gg A$

$$
\Delta S=-\frac{8}{3} \pi^{2} \alpha_{H}^{2} \frac{A^{2}}{l^{2}} .
$$

It is useful to note that, for given $(l, t)$, the minimal surfaces in eq. (5.9) in the Poincaré patch are mapped by eq. (3.3) to constant $\tau$ surfaces in global AdS. These surfaces are attached at $r \rightarrow \infty$ to a circle with constant $\theta=\theta_{0}$, where

$$
\theta_{0}(l, t)=\tan ^{-1}\left(\frac{2 A l}{l^{2}-t^{2}-A^{2}}\right),
$$

which corresponds to a parallel on the $S^{2}$ boundary. This shows that $\Delta S(l, t)$ is a function just of the combination in eq. (5.19).

The RT surfaces with

$$
l=l_{0}=\sqrt{t^{2}+A^{2}}
$$

corresponds to a parallel with $\theta_{0}=\pi / 2$. These surfaces are special, because they lie on the equator of $S^{2}$. Due to symmetry, we conclude that the RT surface at $l=l_{0}$ has either the maximal or the minimal $\Delta S$. For the monopole case, we know that $\Delta S$ is negative and close to zero for $t \rightarrow 0$ and large $l$. So we expect that $l=l_{0}$ is a minimum of $\Delta S$, as also confirmed by the numerics in figure 9 .

For $l=l_{0}, \Delta S$ can be computed exactly:

$$
\Delta S_{0}=\Delta S\left(l_{0}\right)=\frac{\pi^{2} \alpha_{H}^{2}}{4} \int_{0}^{\infty}\left(h_{\epsilon}-g_{\epsilon}\right) \frac{r}{\sqrt{1+r^{2}}} d r=-\Upsilon \frac{\pi^{2} \alpha_{H}^{2}}{4},
$$

where

$$
\Upsilon=6 \pi-12-8 \pi \beta(2)+14 \zeta(3) \approx 0.658 .
$$

In this expression, $\beta(2) \approx 0.916$ is the Catalan constant and $\zeta$ is the Riemann zeta function.

Summarising, the entropy of the disk with radius $l_{0}=\sqrt{t^{2}+A^{2}}$ remains constant as a function of the time $t$ and equal to the minimum $\Delta S_{0}$. This can be heuristically justified as follows. At large $t$, the bound from causality on the speed of entanglement propagation is saturated: $\Delta S$, which originated at $t=0$ from a region nearby $x=0$, spreads at the speed of light. At small $t$, the speed of propagation is smaller, because at $t \rightarrow 0$ also the matter of the quench has zero velocity: entanglement spreads with matter. 

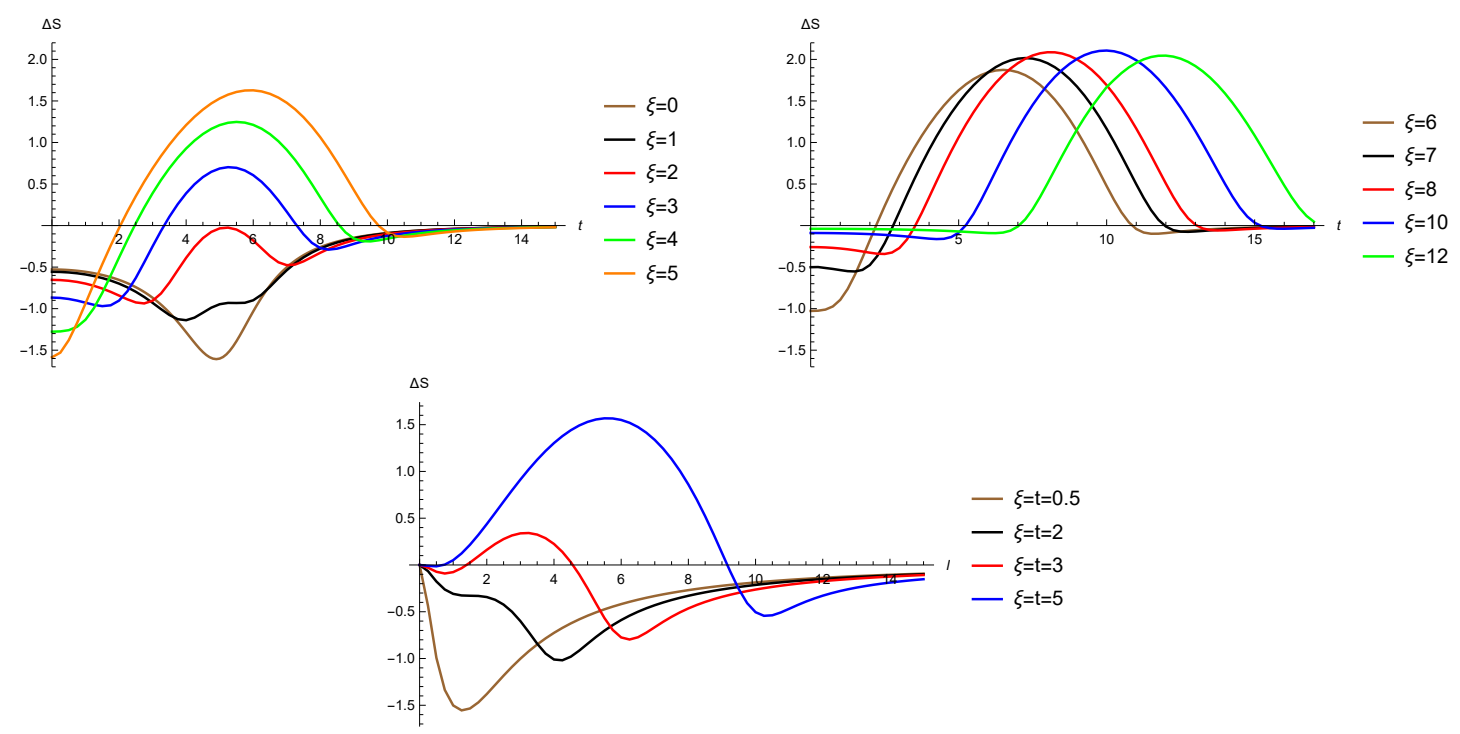

Figure 10: Left and right: time dependence of $\Delta S$ for a disk-shaped subregion of radius $l=5$ centered at $\left(x_{1}, x_{2}\right)=(\xi, 0)$ for different values of $\xi$. For large $\xi$, the maximum is reached for $t \approx \xi$. Bottom: plot of $\Delta S$ as a function of $l$ for a translated disk-shaped subregion, for various values of $t=\xi$. Numerical values $\alpha_{H}=1, A=1$ have been fixed.

\subsection{Translated disk}

For convenience, we introduce

$$
\vec{x}=\left(x_{1}, x_{2}\right)=(x \cos \varphi, x \sin \varphi) .
$$

We now consider as subregion a disk of radius $l$ centered at $\left(x_{1}, x_{2}\right)=(\xi, 0)$ and lying at constant time $t$. The corresponding RT surface in unperturbed Poincaré $\mathrm{AdS}_{4}$ is the translated half sphere

$$
z=\sqrt{l^{2}-\left(x_{1}-\xi\right)^{2}-x_{2}^{2}} .
$$

In appendix D.1 we write the explicit integral for the first-order correction to the holographic entanglement entropy. It is convenient to rescale spatial and time coordinates as in eq. (5.12), with $\xi \rightarrow \xi / A$ as well. Numerical results can be obtained for arbitrary radius $l$, see figure 10 .

In the regime of small $l$, the RT surface stays at large $r$, so we can use the expansion (5.13) and a compact expression can be found

$$
\Delta S(l, t, \xi)=-\frac{8}{3} \pi^{2} \alpha_{H}^{2} \frac{A^{2} l^{2}}{A^{4}+2 A^{2}\left(\xi^{2}+t^{2}\right)+\left(t^{2}-\xi^{2}\right)^{2}} .
$$

This shows that $\Delta S$ is always negative for disks with small radius $l$. The entanglement entropy of small disks is then dominated by the negative contribution due to the scalar condensation.

At large $l \gg \xi$, the subregion is, with good approximation, a disk centered at $\xi \approx 0$, and so, from the results of the previous section, we expect a negative $\Delta S$. For intermediate 


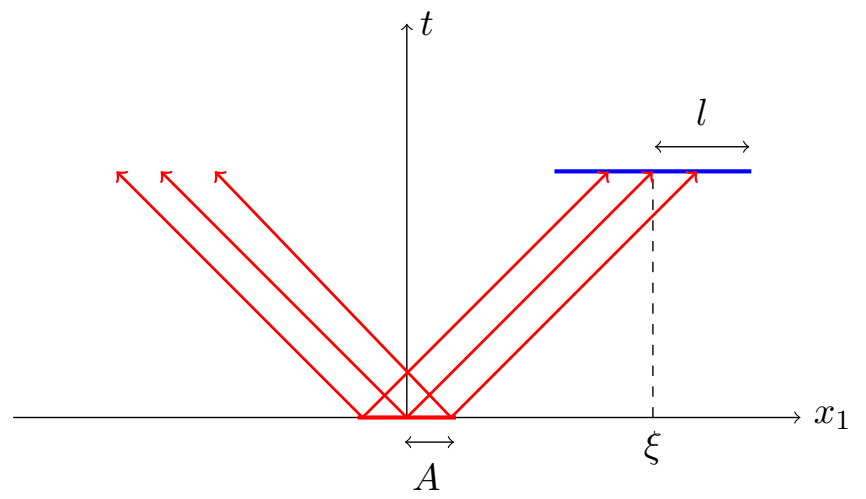

Figure 11: In the quasiparticle model, the quench creates EPR pairs of entangled quasiparticles which subsequently propagate without interactions. When just one of the quasiparticles belonging to an EPR pair is inside the blue region, the entanglement entropy of the region increase.

$l$, the quantity $\Delta S$ can become positive, see figure 10. In this regime we can interpret the positive contribution to $\Delta S$ as due to quasiparticles.

Free quasiparticles provide a simple model of entanglement propagation [9]. In this picture, the quench is assumed to create many copies of Einstein-Podolsky-Rosen (EPR) pairs, which then propagate without interactions, see figure 11. When just one of the entangled particles in an EPR pair is inside a given region, there is a positive contribution to the entanglement entropy. This model can reproduce several aspects of the spread of entanglement in global and local quenches. Models with interacting quasiparticles have also been studied [20]. In all these models, the contribution of the excitations to the entanglement entropy is always positive. In the monopole quench, there is also a negative contribution to the entanglement entropy due to the scalar condensate. In general, we expect that there is a competition between the quasiparticle and the condensate contribution, which is responsible for the change of sign of the entanglement entropy of the translated disk region.

\subsection{Half-plane region}

We take as a boundary subregion the half-plane $x_{1} \geq 0$ at constant time $t$. The unperturbed $\mathrm{RT}$ is the bulk surface at $x_{1}=0$ and constant time $t$. A convenient choice of parameters is

$$
y^{\alpha}=\left(z, x_{2}\right) .
$$

Details of the calculations are in appendix D.2. From the closed-form expression, we deduce that the entropy variation $\Delta S$ is a function of $t / A$. Numerical result is shown in figure 12 .

For $t=0$, the entropy is given by $\Delta S_{0}$ in eq. (5.21). This is because, due to the change of variables in eq. (3.3), the $t=0$ plane with $x_{1}=0$ (which corresponds to $\varphi= \pm \pi / 2$ ) is mapped in global AdS to a disk with $\tau=0$ and constant $\varphi= \pm \pi / 2$. Then, an explicit computation easily leads to the same entropy as in (5.21). 


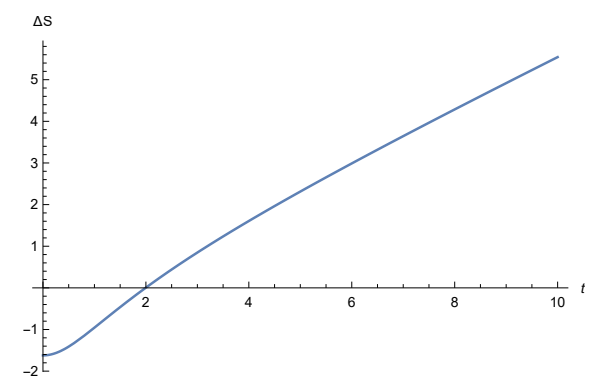

Figure 12: Time dependence of $\Delta S$ for the half-plane subregion. The numerical values $\alpha_{H}=1, A=1$ have been chosen.

At large $t$, from the analysis in appendix D.2, we find that $\Delta S$ scales in a linear way with time, i.e.

$$
\Delta S=K \alpha_{H}^{2} \frac{t}{A}, \quad K \approx 0.636 .
$$

An exact expression for $K$ is given in eq. (D.13). This is consistent with numerical results, shown in figure 12. We emphasise that this result is valid only in the regime where we can trust our perturbative calculation in the parameter $\epsilon$. At very large $t$, we expect that the large backreaction effects spoil the results in eq. (5.27). A linear behaviour at large $t$ is also realised for the perturbative entropy in the case of a falling black hole in $\mathrm{AdS}_{4}$ [38].

The numerical plot in figure 12 is consistent with both the $t=0$ and large $t$ analytical calculations. At $t=0, \Delta S$ is negative, in agreement with the expectation that the condensate decreases the entanglement entropy. After an initial transient time, the quantity $\Delta S$ enters in a linear growth regime and becomes positive around $t \approx 2 A$. The asymptotic linear behaviour is similar to the one of the black hole quench and we expect that it is due to the contribution of quasiparticles.

\subsection{Comparison with the $\mathrm{BH}$ quench}

In the case of a falling black hole in $\mathrm{AdS}_{4}$, the perturbative entropy for a centered disk is

$$
\Delta S^{(B H)}=\frac{\pi M L^{2}}{4 G A l}\left(\frac{l^{4}-2 l^{2} t^{2}+\left(A^{2}+t^{2}\right)^{2}}{\sqrt{l^{4}+2 l^{2}\left(A^{2}-t^{2}\right)+\left(A^{2}+t^{2}\right)^{2}}}-\left|t^{2}+A^{2}-l^{2}\right|\right),
$$

where $M$ is the mass parameter, as defined in eq. (4.18). In this case, $\Delta S^{(B H)}$ is always positive and has a maximum for $l=l_{0}=\sqrt{t^{2}+A^{2}}$. The First Law of Entanglement Entropy (FLEE) [63] is valid in the regime of small $l$ :

$$
\Delta S=\frac{\Delta E}{T_{E}}, \quad T_{E}=\frac{2}{\pi l},
$$

where $T_{E}$ is the entanglement temperature and $\Delta E$ is the energy.

FLEE is generically invalidated in the case of backgrounds with scalars, because the Fefferman-Graham expansion of the metric does not start at order $z^{d}[64,65]$, where $d$ is the dimension of the spacetime boundary. Indeed, as can be checked from eq. (5.25), FLEE 
is not satisfied for the monopole quench background. The behaviour of $\Delta S$ for small $l$ in eq. (5.25) is rather different from the FLEE regime. In particular:

- $\Delta S$ is negative;

- the quantity $\Delta S$ scales as $l^{2}$, and not as $l^{3}$ as predicted by FLEE;

- there is no choice of boundary conditions for which the energy $\Delta E$ is proportional to $\Delta S$.

The FLEE can be derived from the notion of relative entropy $[6,64]$, which is a quantity that measures how far is a density matrix $\rho$ from a reference density matrix $\sigma$ :

$$
S(\rho \| \sigma)=\operatorname{Tr}(\rho \log \rho)-\operatorname{Tr}(\rho \log \sigma) .
$$

As a general property, $S(\rho \| \sigma)$ is positive definite and it vanishes if and only if $\rho=\sigma$. The relative entropy can be written as

$$
S(\rho \| \sigma)=\Delta\left\langle\mathcal{K}_{\sigma}\right\rangle-\Delta S
$$

where $\mathcal{K}_{\sigma}$ is the modular Hamiltonian of the density matrix $\sigma$

$$
\mathcal{K}_{\sigma}=-\log \sigma .
$$

From positivity of relative entropy we get the relation

$$
\Delta\left\langle\mathcal{K}_{\sigma}\right\rangle \geq \Delta S
$$

The modular Hamiltonian operator $\mathcal{K}_{\sigma}$ for a spherical domain with radius $l$ in the vacuum state of a $d$-dimensional CFT can be expressed [66] in terms of the energy-momentum operator as follows

$$
\mathcal{K}_{\sigma}=2 \pi \int_{\text {sphere }} d^{d-1} \vec{x} \frac{l^{2}-x^{2}}{2 l} T_{t t}(\vec{x}),
$$

where $x=|\vec{x}|$. In the limit of small spherical subregion and in $d=3$, we find

$$
\Delta\left\langle\mathcal{K}_{\sigma}\right\rangle=\frac{\pi l}{2} \Delta E,
$$

so the FLEE in eq. (5.29) follows from the saturation of the identity (5.33), i.e.

$$
\Delta\left\langle\mathcal{K}_{\sigma}\right\rangle=\Delta S .
$$

If we consider two nearby density matrices, i.e.

$$
\sigma=\rho_{0}, \quad \rho=\rho_{0}+\varepsilon \rho_{1}+O\left(\varepsilon^{2}\right),
$$

where $\varepsilon$ is an expansion parameter, the relative entropy scales with $\varepsilon$ as follows

$$
S(\rho \| \sigma)=O\left(\varepsilon^{2}\right) .
$$


Since the $O(\varepsilon)$ contribution to relative entropy vanishes, there is a general expectation [64] that for small deformations eq. (5.33) is saturated. However, the question if FLEE is satisfied in quantum field theory is subtle: there the density matrix $\rho$ is infinite dimensional and so it is not clear, in principle, when a perturbation might be considered small.

For a fixed value of the multitrace coupling $\kappa$, the monopole solution can not be continuously deformed to the vacuum due to topological arguments. For this reason, eq. (5.37) can not be realised for arbitrary small $\varepsilon$. Then, the monopole deformation can not be considered close enough to the vacuum and FLEE is not satisfied.

\section{Conclusions}

In this paper, we studied a magnetic monopole solution in the static global $\mathrm{AdS}_{4}$ setup introduced in [51]. We found an approximate analytical solution for the monopole in the regime of small gauge coupling $e$ which includes the leading-order backreaction on the metric. By using the map introduced in [52], the static monopole in global $\mathrm{AdS}_{4}$ is mapped into a falling monopole in the Poincaré patch. This bulk configuration is dual to a local quench on the CFT side. The expectation values of local operators depend on the choice of boundary conditions. With Dirichlet or Neumann conditions, the falling monopole is dual to a field theory with a time-dependent source. With the special choice of multitrace deformation in eq. (4.14), the monopole is dual to a field theory with zero sources. In this case, there is no energy injection and the form of the energy-momentum tensor is the same as the one of a falling black hole [31].

Then, we computed the entanglement entropy due to the leading order classical bulk backreaction. We focused on a regime where the monopole mass in eq. (4.30) is sufficiently large in order to neglect quantum corrections, see eqs. (5.2). The behaviour of entanglement entropy is rather different compared to the case of the falling black hole. For spherical regions centered on the local quench, the perturbed entanglement entropy is always less than the vacuum value, i.e. $\Delta S \leq 0$. This is consistent with the presence of a condensate at the core of the local quench [53].

In the case of a spherical region not centered at the origin, there is a competition in $\Delta S$ between the negative contribution from the condensate and the positive one due to quasiparticles [9]. Depending on the radius $l$ and on the distance $\xi$ from the origin of the spherical region, $\Delta S$ can be positive or negative, see figure 10. In the case of half-plane region, the negative contribution to $\Delta S$ due to the condensate dominates at early times, while the positive contribution due to quasiparticles dominates at late times (see figure 12).

For a quench dual to a falling black hole, the First Law of Entanglement Entropy (FLEE) [63] is satisfied for small subregions. For the quench dual to the monopole, we found that the FLEE is not satisfied. This is a feature shared with other AdS backgrounds which involve the backreaction of scalar bulk fields, see [64, 65]. On the field theory side, the violation of the FLEE comes from the non-saturation of the inequality in eq. (5.33). It is still an open question whether a given deformation obeys or not the FLEE [65] in a quantum field theory. It would be interesting to further investigate FLEE in non-equilibrium systems, in order to understand its general validity conditions. 
Analytical soliton solutions which include backreaction are quite rare in AdS spacetime. The monopole solution found in this paper can be the starting point for several further investigations. In particular, it would be interesting to study more general solitonic objects in AdS. For instance, vortex strings in AdS were considered by many authors [67-75]. A static configuration in Poincaré patch with a monopole attached to a vortex string should also be possible, as proposed in [72]: the vortex string can pull the vortex and counterbalance the gravitational force that makes it fall towards the center of AdS. It would be interesting to find explicit solutions for these objects and to investigate their field theory duals.

Another possible direction is the study of holographic complexity [76-78]. Quantum computational complexity is a recent quantum information entry in the holographic dictionary, which was motivated by the desire of understanding the growth of the Einstein-Rosen bridge inside the event horizon of black holes. Complexity for several examples of global and local quenches has been studied by several authors, e.g. [79-87]. It would be interesting to investigate complexity for quenches dual to a falling monopole. This analysis may give us useful insights to understand the impact of condensates on quantum complexity.

\section{Acknowledgments}

We are grateful to Stefano Bolognesi for useful discussions. N.Z. acknowledges the Ermenegildo Zegna's Group for the financial support.

\section{A Equations of motion}

\section{A.1 Coordinate $r$}

In the probe approximation, the equations of motion are

$$
\begin{aligned}
& F^{\prime \prime}=-F^{\prime} \frac{2\left(1+2 r^{2}\right)}{r\left(1+r^{2}\right)}-F \frac{-2+2 r^{2}+3 e r F-e^{2} r^{2} F^{2}}{r^{2}\left(1+r^{2}\right)}-H^{2} \frac{e(1-e r F)}{r\left(1+r^{2}\right)}, \\
& H^{\prime \prime}=-H^{\prime} \frac{2\left(1+2 r^{2}\right)}{r\left(1+r^{2}\right)}+2 H \frac{(1-e r F)^{2}}{r^{2}\left(1+r^{2}\right)}-\frac{2 H}{1+r^{2}} .
\end{aligned}
$$

Including backreaction, the full set of equations of motion is

$$
\begin{aligned}
F^{\prime \prime}= & -F^{\prime} \frac{2\left(1+2 r^{2}\right)}{r\left(1+r^{2}\right)}-\frac{g^{\prime}}{g}\left(\frac{F}{r}+F^{\prime}\right)-H^{2} \frac{e(1-e r F)}{r\left(1+r^{2}\right)} \frac{h}{g} \\
& -F \frac{1}{r^{2}\left(1+r^{2}\right)}\left(2 r^{2}+\frac{h}{g}\left[-2+3 e r F-e^{2} r^{2} F^{2}\right]\right) \\
H^{\prime \prime}= & -H^{\prime}\left(\frac{2\left(1+2 r^{2}\right)}{r\left(1+r^{2}\right)}+\frac{g^{\prime}}{g}\right)-\frac{h}{g}\left(-2 H \frac{(1-e r F)^{2}}{r^{2}\left(1+r^{2}\right)}+\frac{2 H}{1+r^{2}}\right), \\
g^{\prime}= & \frac{4 \pi G}{L^{2}} h \frac{2 H^{2}\left[(2-e r F) e r F+r^{2}-1\right]-F^{2}(2-e r F)^{2}}{r\left(1+r^{2}\right)}+\frac{1+3 r^{2}}{r\left(r^{2}+1\right)}(h-g), \\
h^{\prime}= & \frac{8 \pi G}{L^{2}} h\left(\frac{r}{2}\left(H^{\prime}\right)^{2}+\frac{\left(F+r F^{\prime}\right)^{2}}{r}\right) .
\end{aligned}
$$




\section{A.2 Coordinate $\psi$}

In the probe approximation, we find

$$
\begin{aligned}
F^{\prime \prime} & =\frac{e^{2} F^{3}+F\left(e^{2} H^{2}+2 \cot ^{2}(\psi)-2\right)-3 e F^{2} \cot (\psi)-\cot (\psi)\left(e H^{2}+2 F^{\prime}\right)}{\cos ^{2} \psi}, \\
H^{\prime \prime} & =4 \frac{H\left(e^{2} F^{2} \tan (\psi)-2 e F+2 \cot (2 \psi)\right)-H^{\prime}}{\sin (2 \psi)} .
\end{aligned}
$$

Including backreaction, we get

$$
\begin{aligned}
F^{\prime \prime}= & \frac{1}{g}\left[-e \csc \psi \sec \psi\left(3 F^{2}+H^{2}\right) h+e^{2} \sec ^{2} \psi F^{3} h-F^{\prime}\left(2 g \csc \psi \sec \psi+g^{\prime}\right)\right. \\
& \left.+F\left(h\left(2 \csc ^{2} \psi+e^{2} H^{2} \sec ^{2} \psi\right)-\sec \psi\left(2 g \sec \psi+g^{\prime} \csc \psi\right)\right)\right], \\
H^{\prime \prime}= & \frac{1}{g}\left[2 h H\left(\csc ^{2} \psi-2 e F \csc \psi \sec \psi+\sec ^{2} \psi\left(-1+e^{2} F^{2}\right)\right)\right. \\
& \left.-\left(2 g \csc \psi \sec \psi+g^{\prime}\right) H^{\prime}\right], \\
h^{\prime}= & \frac{2 \pi G}{L^{2}} h\left[2\left(F \csc \psi \sec \psi+F^{\prime}\right)^{2}+H^{\prime 2}\right] \sin 2 \psi, \\
g^{\prime}= & \tan \psi\left(3+\cot ^{2} \psi\right)(h-g)+\frac{4 \pi G}{L^{2}} h \tan \psi\left[-F^{2}(-2 \cot \psi+e F)^{2}\right. \\
& \left.+2 H^{2}-2(\cot \psi-e F)^{2} H^{2}\right] .
\end{aligned}
$$

\section{B Abelian field strength and flux}

The abelian field strength and its dual are [40]:

$$
\mathcal{F}_{\mu \nu}=n^{a} F_{\mu \nu}^{a}-\frac{1}{e} \epsilon^{a b c} n^{a} D_{\mu} n^{b} D_{\nu} n^{c}, \quad \tilde{\mathcal{F}}^{\mu \nu} \equiv \frac{1}{2} \frac{\epsilon^{\mu \nu \alpha \beta}}{\sqrt{-g}} \mathcal{F}_{\alpha \beta},
$$

which satisfies

$$
D_{\mu} \tilde{\mathcal{F}}^{\mu \nu}=\frac{4 \pi}{e} k^{\nu}, \quad k_{\mu}=\frac{1}{8 \pi} \epsilon_{\mu \nu \rho \sigma} \epsilon_{a b c} \partial^{\nu} n^{a} \partial^{\rho} n^{b} \partial^{\sigma} n^{c},
$$

where $k_{\mu}$ is the topological current. The only non-vanishing components of the dual electromagnetic tensor $\tilde{\mathcal{F}}^{\mu \nu}$ are:

$$
\tilde{\mathcal{F}}^{t r}=-\tilde{\mathcal{F}}^{r t}=-\frac{1}{e r^{2}} .
$$

The magnetic flux on a sphere of radius $r$ is given by Stokes theorem:

$$
Q=\int_{S^{2}} \tilde{\mathcal{F}}^{\mu \nu} d S_{\mu \nu}, \quad d S_{\mu \nu}=n_{[\mu} r_{\nu]} r^{2} \sin \theta d \theta d \varphi
$$

where $n_{\mu}$ and $r_{\nu}$ are the unit time and radial vectors, respectively. A direct computation gives:

$$
Q=-\frac{8 \pi}{e} .
$$

Note that the magnetic flux is topological and independent of the profile functions details. 


\section{Details of the boundary energy-momentum tensor}

\section{C.1 Black hole}

In Fefferman-Graham (FG) coordinates, the metric has the following form

$$
\hat{x}^{\mu}=(\hat{z}, \hat{t}, \hat{x}, \hat{\varphi}) \quad d s^{2}=L^{2}\left(\frac{d \hat{z}^{2}}{\hat{z}^{2}}+\frac{1}{\hat{z}^{2}} g_{a b}\left(\hat{z}, \hat{x}^{a}\right) d \hat{x}^{a} d \hat{x}^{b}\right),
$$

where the index $a$ runs on boundary coordinates

$$
\hat{x}^{a}=(\hat{t}, \hat{x}, \hat{\varphi}),
$$

and we take $\hat{\varphi}=\varphi$. The FG coordinates can be built in a perturbative way nearby the boundary, i.e.

$$
z=\hat{z}+\sum_{k=2}^{\infty} a_{k}(\hat{x}, \hat{t}) \hat{z}^{k}, \quad x=\hat{x}+\sum_{k=1}^{\infty} b_{k}(\hat{x}, \hat{t}) \hat{z}^{k}, \quad t=\hat{t}+\sum_{k=1}^{\infty} c_{k}(\hat{x}, \hat{t}) \hat{z}^{k} .
$$

Plugging into the metric in Poincaré coordinates and comparing with the FG metric order by order, we get:

$$
\begin{aligned}
& b_{1}=c_{1}=0, \quad a_{2}=b_{2}=c_{2}=0, \quad a_{3}=b_{3}=c_{3}=0 \quad b_{4}=c_{4}=0, \\
& a_{4}=-\frac{4 A^{3} M}{3\left(A^{4}+2 A^{2} \hat{t}^{2}+2 A^{2} \hat{x}^{2}+\hat{t}^{4}-2 \hat{t}^{2} \hat{x}^{2}+\hat{x}^{4}\right)^{3 / 2}} .
\end{aligned}
$$

The energy-momentum tensor can be obtained from the results of [59]

$$
T_{m n}^{(B H)}=\frac{L}{8 \pi G} \lim _{\hat{z} \rightarrow 0} \frac{1}{\hat{z}}\left(K_{m n}-\gamma_{m n} K-\frac{2}{L} \gamma_{m n}\right) .
$$

Here $\gamma_{m n}$ is the induced metric on a $\hat{z}$-constant surface nearby the boundary, $K_{m n}$ denotes the extrinsic curvature tensor calculated with an inward unit vector normal to the $\hat{z}$ constant surface, and $K$ is the trace of the extrinsic curvature tensor.

To explicitly write the components of the energy-momentum tensor, it is convenient to introduce the lightcone coordinates:

$$
u^{m}=(u, v, \varphi), \quad u=t-x, \quad v=t+x .
$$

In these coordinates, the non-vanishing elements of $T_{m n}^{(B H)}$ are

$$
\begin{aligned}
& T_{u u}^{(B H)}=\frac{A^{3} L^{2} M}{8 \pi G} \frac{3}{\left(A^{2}+u^{2}\right)^{5 / 2}\left(A^{2}+v^{2}\right)^{1 / 2}}, \\
& T_{v v}^{(B H)}=\frac{A^{3} L^{2} M}{8 \pi G} \frac{3}{\left(A^{2}+v^{2}\right)^{5 / 2}\left(A^{2}+u^{2}\right)^{1 / 2}}, \\
& T_{u v}^{(B H)}=\frac{A^{3} L^{2} M}{8 \pi G} \frac{1}{\left(A^{2}+u^{2}\right)^{3 / 2}\left(A^{2}+v^{2}\right)^{3 / 2}}, \\
& T_{\varphi \varphi}^{(B H)}=\frac{A^{3} L^{2} M}{8 \pi G} \frac{(u-v)^{2}}{\left(A^{2}+u^{2}\right)^{3 / 2}\left(A^{2}+v^{2}\right)^{3 / 2}} .
\end{aligned}
$$




\section{C.2 Monopole with Dirichlet boundary conditions}

In order to put the metric with monopole backreaction in FG coordinates, we consider the expansion of $h$ and $g$ nearby the boundary in eq. (2.23), setting also $h_{2}=-g_{2}$. Then, using the change of variables in eq. (C.3) and solving order by order, we obtain

$$
\begin{aligned}
& b_{1}=c_{1}=0, \quad a_{2}=b_{2}=c_{2}=0, \quad b_{3}=c_{3}=0, \\
& a_{3}=\frac{2 A^{2} g_{2}}{A^{4}+2 A^{2} \hat{t}^{2}+2 A^{2} \hat{x}^{2}+\hat{t}^{4}-2 \hat{t}^{2} \hat{x}^{2}+\hat{x}^{4}}, \\
& a_{4}=\frac{4 A^{3}\left(g_{3}-h_{3}\right)}{3\left(A^{4}+2 A^{2} \hat{t}^{2}+2 A^{2} \hat{x}^{2}+\hat{t}^{4}-2 \hat{t}^{2} \hat{x}^{2}+\hat{x}^{4}\right)^{3 / 2}}, \\
& b_{4}=-\frac{2 A^{2} g_{2} \hat{x}\left(A^{2}-\hat{t}^{2}+\hat{x}^{2}\right)}{\left(A^{4}+2 A^{2} \hat{t}^{2}+2 A^{2} \hat{x}^{2}+\hat{t}^{4}-2 \hat{t}^{2} \hat{x}^{2}+\hat{x}^{4}\right)^{2}}, \\
& c_{4}=\frac{2 A^{2} g_{2} \hat{t}\left(A^{2}+\hat{t}^{2}-\hat{x}^{2}\right)}{\left(A^{4}+2 A^{2} \hat{t}^{2}+2 A^{2} \hat{x}^{2}+\hat{t}^{4}-2 \hat{t}^{2} \hat{x}^{2}+\hat{x}^{4}\right)^{2}} .
\end{aligned}
$$

We can now use the generalisation of eq. (C.5) involving scalars [60] to extract the energymomentum tensor

$$
T_{m n}^{(D)}=\frac{L}{8 \pi G} \lim _{\hat{z} \rightarrow 0} \frac{1}{\hat{z}}\left(K_{m n}-\gamma_{m n} K-\frac{2}{L} \gamma_{m n}-4 \pi G \frac{\gamma_{m n}}{L} \phi^{a} \phi^{a}\right) .
$$

The elements of the energy-momentum tensor in lightcone coordinates look qualitatively similar to the corresponding elements computed in the BH background, see eq. (C.7):

$$
\begin{aligned}
& T_{u u}^{(D)}=T_{u u}^{(B H)}\left(\frac{16 \pi G \alpha_{H} \beta_{H}-3 L^{2} g_{3}}{3 L^{2} M}\right), \\
& T_{v v}^{(D)}=T_{v v}^{(B H)}\left(\frac{16 \pi G \alpha_{H} \beta_{H}-3 L^{2} g_{3}}{3 L^{2} M}\right), \\
& T_{u v}^{(D)}=T_{u v}^{(B H)}\left(\frac{-16 \pi G \alpha_{H} \beta_{H}-3 L^{2} g_{3}}{3 L^{2} M}\right), \\
& T_{\varphi \varphi}^{(D)}=T_{\varphi \varphi}^{(B H)}\left(\frac{32 \pi G \alpha_{H} \beta_{H}-3 L^{2} g_{3}}{3 L^{2} M}\right) .
\end{aligned}
$$

The Ward identity for $T_{m n}^{(D)}$ gives

$$
\partial^{m} T_{m n}^{(D)}=\tilde{\beta}_{H} \partial_{n} \tilde{\alpha}_{H}=\left\langle\mathcal{O}_{2}\right\rangle \partial_{n} J_{D},
$$

and the trace of the energy-momentum tensor is

$$
\eta^{m n} T_{m n}^{(D)}=\tilde{\alpha}_{H} \tilde{\beta}_{H}=\left\langle\mathcal{O}_{2}\right\rangle J_{D}
$$




\section{C.3 Monopole with Neumann and multitrace boundary conditions}

We will follow the approach in [58] to determine the boundary energy-momentum tensor for multitrace deformations. These boundary conditions correspond to adding to the renormalised action $S_{\text {ren }}$ a finite boundary action $S_{\mathcal{F}}$ given by

$$
S_{\mathcal{F}}=\int d^{3} x \sqrt{-g_{0}}\left(J_{F} \tilde{\alpha}_{H}+\mathcal{F}\left(\tilde{\alpha}_{H}\right)\right), \quad J_{\mathcal{F}}=-\tilde{\beta}_{H}-\mathcal{F}^{\prime}\left(\tilde{\alpha}_{H}\right)
$$

where $\sqrt{-g_{0}}$ is the determinant of the boundary metric (at the end of the calculation we will specialise to the Minkowski metric). The variations of the action functionals are:

$$
\begin{aligned}
\delta S_{\mathrm{ren}} & =\int d^{3} x \sqrt{-g_{0}}\left(\frac{1}{2} T^{i j}\left(\delta g_{(0)}\right)_{i j}+\tilde{\beta}_{H} \delta \tilde{\alpha}_{H}\right) \\
\delta S_{\mathcal{F}} & =\int d^{3} x \sqrt{-g_{0}}\left(-\tilde{\alpha}_{H} \delta \tilde{\beta}_{H}-\tilde{\alpha}_{H} \mathcal{F}^{\prime \prime}\left(\tilde{\alpha}_{H}\right) \delta \tilde{\alpha}_{H}-\tilde{\beta}_{H} \delta \tilde{\alpha}_{H}\right)
\end{aligned}
$$

so the total variation is

$$
\begin{aligned}
\delta S & =\delta S_{\text {ren }}+\delta S_{\mathcal{F}}=\int d^{3} x \sqrt{-g_{0}}\left(\frac{1}{2} T^{i j}\left(\delta g_{(0)}\right)_{i j}-\tilde{\alpha}_{H} \delta \tilde{\beta}_{H}-\tilde{\alpha}_{H} \mathcal{F}^{\prime \prime}\left(\tilde{\alpha}_{H}\right) \delta \tilde{\alpha}_{H}\right) \\
& =\int d^{3} x \sqrt{-g_{0}}\left(\frac{1}{2} T^{i j}\left(\delta g_{(0)}\right)_{i j}+\tilde{\alpha}_{H} \delta J_{\mathcal{F}}\right) .
\end{aligned}
$$

Due to the shift of the action, there is also a shift in the energy-momentum tensor:

$$
T_{i j}^{(\mathcal{F})}=T_{i j}^{(D)}+\eta_{i j}\left[\mathcal{F}\left(\tilde{\alpha}_{H}\right)+\tilde{\alpha}_{H} J_{F}\right]=T_{i j}^{(D)}+\eta_{i j}\left[\mathcal{F}\left(\tilde{\alpha}_{H}\right)-\tilde{\alpha}_{H} \tilde{\beta}_{H}-\mathcal{F}^{\prime}\left(\tilde{\alpha}_{H}\right) \tilde{\alpha}_{H}\right] .
$$

The divergence of the energy-momentum tensor is:

$$
\begin{aligned}
\partial^{i} T_{i j}^{(\mathcal{F})} & =\partial^{i} T_{i j}^{(D)}+\partial_{j}\left[\mathcal{F}\left(\tilde{\alpha}_{H}\right)-\tilde{\alpha}_{H} \tilde{\beta}_{H}-\mathcal{F}^{\prime}\left(\tilde{\alpha}_{H}\right) \tilde{\alpha}_{H}\right] \\
& =\tilde{\beta}_{H} \partial_{j} \tilde{\alpha}_{H}+\partial_{j}\left[\mathcal{F}\left(\tilde{\alpha}_{H}\right)-\tilde{\alpha}_{H} \tilde{\beta}_{H}-\mathcal{F}^{\prime}\left(\tilde{\alpha}_{H}\right) \tilde{\alpha}_{H}\right] \\
& =-\tilde{\alpha}_{H}\left(\partial_{j} \tilde{\beta}_{H}+\mathcal{F}^{\prime \prime}\left(\tilde{\alpha}_{H}\right) \partial_{j} \tilde{\alpha}_{H}\right),
\end{aligned}
$$

while the trace is:

$$
\begin{aligned}
\eta^{i j} T_{i j}^{(\mathcal{F})} & =\left(T^{(D)}\right)_{i}^{i}+3\left[\mathcal{F}\left(\tilde{\alpha}_{H}\right)-\tilde{\alpha}_{H} \tilde{\beta}_{H}-\mathcal{F}^{\prime}\left(\tilde{\alpha}_{H}\right) \tilde{\alpha}_{H}\right] \\
& =-2 \tilde{\alpha}_{H} \tilde{\beta}_{H}+3 \mathcal{F}\left(\tilde{\alpha}_{H}\right)-3 \tilde{\alpha}_{H} \mathcal{F}^{\prime}\left(\tilde{\alpha}_{H}\right) .
\end{aligned}
$$

Setting to zero the source $J_{\mathcal{F}}$ corresponds to

$$
\tilde{\beta}_{H}=-\mathcal{F}^{\prime}\left(\tilde{\alpha}_{H}\right)
$$

Note that in this case $T_{i j}^{(\mathcal{F})}$ is conserved, i.e. $\partial^{i} T_{i j}^{(\mathcal{F})}=0$. 


\section{Details of the entanglement entropy calculations}

\section{D.1 Translated-disk region}

Defining the polar-like coordinates

$$
x_{1}=\xi+p \cos \vartheta, \quad x_{2}=p \sin \vartheta,
$$

the entanglement entropy is given by

$$
\begin{aligned}
\Delta S= & \frac{\pi \alpha_{H}^{2}}{2 l} \int_{0}^{l} d p \frac{p}{\left(l^{2}-p^{2}\right)^{3 / 2}} \int_{0}^{2 \pi} d \vartheta\left[\frac{\left(h_{\varepsilon}+g_{\varepsilon}\right)\left(p^{2} \cos ^{2} \vartheta-l^{2}\right) \xi^{2} t^{2}}{\omega_{\xi}}\right. \\
& +\frac{\left(h_{\varepsilon}-g_{\varepsilon}\right)}{4} \frac{\left[p \omega_{\xi}+2 \xi \cos \vartheta\left(l^{2}-p^{2}\right)\left(l^{2}-t^{2}+A^{2}+\xi^{2}+2 \xi p \cos \vartheta\right)\right]^{2}}{\omega_{\xi}\left[\omega_{\xi}+4 A^{2}\left(p^{2}-l^{2}\right)\right]} \\
& \left.+\frac{\left(h_{\varepsilon}-g_{\varepsilon}\right) l^{2} \xi^{2} \sin ^{2} \vartheta\left(l^{2}-p^{2}\right)\left(l^{2}-t^{2}+A^{2}+\xi^{2}+2 \xi p \cos \vartheta\right)^{2}}{\omega_{\xi}\left[\omega_{\xi}+4 A^{2}\left(p^{2}-l^{2}\right)\right]}\right],
\end{aligned}
$$

where

$$
\omega_{\xi} \equiv \omega\left(\sqrt{l^{2}+\xi^{2}+2 \xi p \cos (2 \vartheta)}, t\right),
$$

and $\omega$ is defined in eq. (4.2). In the above integral, both $h_{\varepsilon}$ and $g_{\varepsilon}$ are functions of

$$
r=\frac{1}{2 A} \sqrt{\frac{\omega_{\xi}+4 A^{2}\left(p^{2}-l^{2}\right)}{l^{2}-p^{2}}} .
$$

\section{D.2 Half-plane region}

As in eq. (5.12), the $A$ dependence can be completely reabsorbed introducing the rescaled quantities

$$
z \rightarrow \frac{z}{A}, \quad x_{2} \rightarrow \frac{x_{2}}{A}, \quad t \rightarrow \frac{t}{A} .
$$

Consequently, without loss of generality, from now on we set $A=1$.

For general $t>0$, we can write a closed-form expression for the entropy variation

$$
\Delta S=\frac{\pi \alpha_{H}^{2}}{8} \int_{0}^{+\infty} d z \int_{-\infty}^{+\infty} d x_{2}\left[\frac{\left(h_{\varepsilon}-g_{\varepsilon}\right)\left(C^{2}+4 z^{2} x_{2}^{2} D^{2}\right)}{z^{2}\left(D^{2}+4 t^{2}\right)^{2}}-4 t^{2} \frac{\left(h_{\varepsilon}+g_{\varepsilon}\right)\left(x_{2}^{2}+z^{2}\right)}{z^{2}\left(D^{2}+4 t^{2}\right)}\right]
$$

with

$$
C \equiv t^{4}-z^{4}-2 t^{2}\left(x_{2}^{2}-1\right)+\left(x_{2}^{2}+1\right)^{2}, \quad D \equiv-t^{2}+x_{2}^{2}+z^{2}+1 .
$$

In the above expression, $h_{\varepsilon}(r)$ and $g_{\varepsilon}(r)$ are the functions defined in eq. (2.26), with

$$
r=\frac{\sqrt{\left(-t^{2}+x_{2}^{2}+z^{2}\right)^{2}+2\left(t^{2}+x_{2}^{2}-z^{2}\right)+1}}{2 z} .
$$

In order to understand the large $t$ behaviour of the entropy, it is convenient to introduce the variables $\rho, \gamma$

$$
z=\rho \cos \gamma, \quad x_{2}=\rho \sin \gamma
$$


At large $t$, the integrand in eq. (D.6) is non-vanishing just in a region at $\rho=t \pm \mu$, with $\mu$ of order 1. For convenience, we can introduce

$$
\rho=t+\delta
$$

It turns out that, at large $t$, the term proportional to $\left(h_{\varepsilon}-g_{\varepsilon}\right)$ in eq. (D.6) is much smaller than the one proportional to $\left(h_{\varepsilon}+g_{\varepsilon}\right)$. Moreover, at large $t$, we can use the approximation

$$
r=\frac{\sqrt{\delta^{2}+\sin ^{2} \gamma}}{\cos \gamma}+O(1 / t) .
$$

At the leading order in $t$, we find

$$
\Delta S=K \alpha_{H}^{2} t
$$

where

$$
K=-\frac{\pi}{2} \int_{0}^{\infty} \frac{r}{1+r^{2}} \mathbb{E}\left(-r^{2}\right)\left[h_{\epsilon}(r)+g_{\epsilon}(r)\right] d r .
$$

$\mathbb{E}$ is the complete elliptic integral, defined as follows

$$
\mathbb{E}(m)=\int_{0}^{\pi / 2} d \gamma \sqrt{1-m \sin ^{2} \gamma}
$$

Numerically, we get the approximate value $K \approx 0.636$.

Open Access. This article is distributed under the terms of the Creative Commons Attribution License (CC-BY 4.0), which permits any use, distribution and reproduction in any medium, provided the original author(s) and source are credited.

\section{References}

[1] J.M. Maldacena, The Large $N$ limit of superconformal field theories and supergravity, Int. J. Theor. Phys. 38 (1999) 1113 [hep-th/9711200] [INSPIRE].

[2] S.S. Gubser, I.R. Klebanov and A.M. Polyakov, Gauge theory correlators from noncritical string theory, Phys. Lett. B 428 (1998) 105 [hep-th/9802109] [InSPIRE].

[3] E. Witten, Anti-de Sitter space and holography, Adv. Theor. Math. Phys. 2 (1998) 253 [hep-th/9802150] [INSPIRE].

[4] S. Ryu and T. Takayanagi, Holographic derivation of entanglement entropy from AdS/CFT, Phys. Rev. Lett. 96 (2006) 181602 [hep-th/0603001] [INSPIRE].

[5] V.E. Hubeny, M. Rangamani and T. Takayanagi, A Covariant holographic entanglement entropy proposal, JHEP 07 (2007) 062 [arXiv: 0705.0016] [INSPIRE].

[6] M. Rangamani and T. Takayanagi, Holographic Entanglement Entropy, vol. 931, Springer (2017), [DOI] [arXiv: 1609.01287] [INSPIRE].

[7] J.D. Bekenstein, Black holes and entropy, Phys. Rev. D 7 (1973) 2333 [InSPIRE].

[8] S.W. Hawking, Particle Creation by Black Holes, Commun. Math. Phys. 43 (1975) 199 [Erratum ibid. 46 (1976) 206] [INSPIRE].

[9] P. Calabrese and J.L. Cardy, Evolution of entanglement entropy in one-dimensional systems, J. Stat. Mech. 0504 (2005) P04010 [cond-mat/0503393] [INSPIRE]. 
[10] J. Abajo-Arrastia, J. Aparicio and E. Lopez, Holographic Evolution of Entanglement Entropy, JHEP 11 (2010) 149 [arXiv:1006.4090] [INSPIRE].

[11] T. Albash and C.V. Johnson, Evolution of Holographic Entanglement Entropy after Thermal and Electromagnetic Quenches, New J. Phys. 13 (2011) 045017 [arXiv:1008.3027] [INSPIRE].

[12] V. Balasubramanian et al., Thermalization of Strongly Coupled Field Theories, Phys. Rev. Lett. 106 (2011) 191601 [arXiv: 1012 .4753] [INSPIRE].

[13] V. Balasubramanian et al., Holographic Thermalization, Phys. Rev. D 84 (2011) 026010 [arXiv: 1103.2683] [INSPIRE].

[14] A. Buchel, L. Lehner and R.C. Myers, Thermal quenches in $N=2^{*}$ plasmas, JHEP 08 (2012) 049 [arXiv: 1206.6785] [INSPIRE].

[15] A. Buchel, L. Lehner, R.C. Myers and A. van Niekerk, Quantum quenches of holographic plasmas, JHEP 05 (2013) 067 [arXiv:1302.2924] [INSPIRE].

[16] R. Auzzi, S. Elitzur, S.B. Gudnason and E. Rabinovici, On periodically driven AdS/CFT, JHEP 11 (2013) 016 [arXiv: 1308.2132] [INSPIRE].

[17] A. Buchel, R.C. Myers and A. van Niekerk, Nonlocal probes of thermalization in holographic quenches with spectral methods, JHEP 02 (2015) 017 [Erratum ibid. 07 (2015) 137] [arXiv: 1410.6201] [INSPIRE].

[18] H. Liu and S.J. Suh, Entanglement Tsunami: Universal Scaling in Holographic Thermalization, Phys. Rev. Lett. 112 (2014) 011601 [arXiv:1305.7244] [INSPIRE].

[19] H. Liu and S.J. Suh, Entanglement growth during thermalization in holographic systems, Phys. Rev. D 89 (2014) 066012 [arXiv:1311.1200] [INSPIRE].

[20] H. Casini, H. Liu and M. Mezei, Spread of entanglement and causality, JHEP 07 (2016) 077 [arXiv: 1509.05044] [INSPIRE].

[21] T. Langen, R. Geiger, M. Kuhnert, B. Rauer and J. Schmiedmayer, Local emergence of thermal correlations in an isolated quantum many-body system, Nature Phys. 9 (2013).

[22] F. Meinert, M.J. Mark, E. Kirilov, K. Lauber, P. Weinmann, A.J. Daley and H. Nagerl, Quantum Quench in an Atomic One-Dimensional Ising Chain, Phys. Rev. Lett. 111 (2013).

[23] I. Klich and L. Levitov, Quantum Noise as an Entanglement Meter, Phys. Rev. Lett. 102 (2009) 100502 [arXiv:0804.1377] [inSPIRE].

[24] J. Cardy, Measuring Entanglement Using Quantum Quenches, Phys. Rev. Lett. 106 (2011) 150404 [arXiv: 1012.5116] [INSPIRE].

[25] P. Calabrese and J. Cardy, Entanglement and correlation functions following a local quench: a conformal field theory approach, J. Stat. Mech. 0710 (2007) P10004 [arXiv:0708.3750] [INSPIRE].

[26] P. Calabrese and J. Cardy, Quantum quenches in $1+1$ dimensional conformal field theories, J. Stat. Mech. 1606 (2016) 064003 [arXiv:1603.02889] [INSPIRE].

[27] M. Nozaki, T. Numasawa and T. Takayanagi, Quantum Entanglement of Local Operators in Conformal Field Theories, Phys. Rev. Lett. 112 (2014) 111602 [arXiv:1401.0539] [INSPIRE].

[28] P. Caputa, M. Nozaki and T. Takayanagi, Entanglement of local operators in large- $N$ conformal field theories, PTEP 2014 (2014) 093B06 [arXiv:1405.5946] [INSPIRE]. 
[29] M. Nozaki, Notes on Quantum Entanglement of Local Operators, JHEP 10 (2014) 147 [arXiv: 1405.5875] [INSPIRE].

[30] C.T. Asplund, A. Bernamonti, F. Galli and T. Hartman, Holographic Entanglement Entropy from 2d CFT: Heavy States and Local Quenches, JHEP 02 (2015) 171 [arXiv:1410.1392] [INSPIRE].

[31] M. Nozaki, T. Numasawa and T. Takayanagi, Holographic Local Quenches and Entanglement Density, JHEP 05 (2013) 080 [arXiv: 1302.5703] [INSPIRE].

[32] C.T. Asplund and A. Bernamonti, Mutual information after a local quench in conformal field theory, Phys. Rev. D 89 (2014) 066015 [arXiv:1311.4173] [INSPIRE].

[33] P. Caputa, J. Simón, A. Štikonas and T. Takayanagi, Quantum Entanglement of Localized Excited States at Finite Temperature, JHEP 01 (2015) 102 [arXiv:1410.2287] [InSPIRE].

[34] M. Rangamani, M. Rozali and A. Vincart-Emard, Dynamics of Holographic Entanglement Entropy Following a Local Quench, JHEP 04 (2016) 069 [arXiv: 1512.03478] [INSPIRE].

[35] J.R. David, S. Khetrapal and S.P. Kumar, Universal corrections to entanglement entropy of local quantum quenches, JHEP 08 (2016) 127 [arXiv: 1605. 05987] [INSPIRE].

[36] C.A. Agón, S.F. Lokhande and J.F. Pedraza, Local quenches, bulk entanglement entropy and a unitary Page curve, JHEP 08 (2020) 152 [arXiv:2004.15010] [INSPIRE].

[37] T. Shimaji, T. Takayanagi and Z. Wei, Holographic Quantum Circuits from Splitting/Joining Local Quenches, JHEP 03 (2019) 165 [arXiv:1812.01176] [INSPIRE].

[38] A. Jahn and T. Takayanagi, Holographic entanglement entropy of local quenches in AdS4/CFT3: a finite-element approach, J. Phys. A 51 (2018) 015401 [arXiv:1705.04705] [INSPIRE].

[39] D.S. Ageev, Sharp disentanglement in holographic charged local quench, arXiv:2003.02918 [INSPIRE].

[40] G. 't Hooft, Magnetic Monopoles in Unified Gauge Theories, Nucl. Phys. B 79 (1974) 276 [INSPIRE].

[41] A.R. Lugo and F.A. Schaposnik, Monopole and dyon solutions in AdS space, Phys. Lett. B 467 (1999) 43 [hep-th/9909226] [INSPIRE].

[42] A.R. Lugo, E.F. Moreno and F.A. Schaposnik, Monopole solutions in AdS space, Phys. Lett. B 473 (2000) 35 [hep-th/9911209] [INSPIRE].

[43] S. Bolognesi and D. Tong, Monopoles and Holography, JHEP 01 (2011) 153 [arXiv: 1010.4178] [INSPIRE].

[44] P. Sutcliffe, Monopoles in AdS, JHEP 08 (2011) 032 [arXiv:1104.1888] [INSPIRE].

[45] S. Bolognesi, J.N. Laia, D. Tong and K. Wong, A Gapless Hard Wall: Magnetic Catalysis in Bulk and Boundary, JHEP 07 (2012) 162 [arXiv: 1204.6029] [INSPIRE].

[46] S. Prem Kumar, A. O'Bannon, A. Pribytok and R. Rodgers, Holographic Coulomb branch solitons, quasinormal modes, and black holes, JHEP 05 (2021) 109 [arXiv:2011.13859] [INSPIRE].

[47] A.R. Lugo, E.F. Moreno and F.A. Schaposnik, Holographic Phase Transition from Dyons in an AdS Black Hole Background, JHEP 03 (2010) 013 [arXiv: 1001.3378] [INSPIRE].

[48] A.R. Lugo, E.F. Moreno and F.A. Schaposnik, Holography and AdS 4 self-gravitating dyons, JHEP 11 (2010) 081 [arXiv: 1007.1482] [inSPIRE]. 
[49] G.L. Giordano and A.R. Lugo, Holographic phase transitions from higgsed, non abelian charged black holes, JHEP 07 (2015) 172 [arXiv:1501.04033] [INSPIRE].

[50] S. Miyashita and K.-i. Maeda, AdS Monopole Black Hole and Phase Transition, Phys. Rev. D 94 (2016) 124037 [arXiv:1610.07350] [INSPIRE].

[51] A. Esposito, S. Garcia-Saenz, A. Nicolis and R. Penco, Conformal solids and holography, JHEP 12 (2017) 113 [arXiv:1708.09391] [INSPIRE].

[52] G.T. Horowitz and N. Itzhaki, Black holes, shock waves, and causality in the AdS/CFT correspondence, JHEP 02 (1999) 010 [hep-th/9901012] [INSPIRE].

[53] T. Albash and C.V. Johnson, Holographic Studies of Entanglement Entropy in Superconductors, JHEP 05 (2012) 079 [arXiv: 1202.2605] [INSPIRE].

[54] E. Witten, Multitrace operators, boundary conditions, and AdS/CFT correspondence, hep-th/0112258 [INSPIRE].

[55] M. Berkooz, A. Sever and A. Shomer, 'Double trace' deformations, boundary conditions and space-time singularities, JHEP 05 (2002) 034 [hep-th/0112264] [INSPIRE].

[56] I. Papadimitriou, Multi-Trace Deformations in AdS/CFT: Exploring the Vacuum Structure of the Deformed CFT, JHEP 05 (2007) 075 [hep-th/0703152] [INSPIRE].

[57] T. Faulkner, G.T. Horowitz and M.M. Roberts, Holographic quantum criticality from multi-trace deformations, JHEP 04 (2011) 051 [arXiv: 1008.1581] [INSPIRE].

[58] M.M. Caldarelli, A. Christodoulou, I. Papadimitriou and K. Skenderis, Phases of planar AdS black holes with axionic charge, JHEP 04 (2017) 001 [arXiv: 1612.07214] [INSPIRE].

[59] V. Balasubramanian and P. Kraus, A Stress tensor for Anti-de Sitter gravity, Commun. Math. Phys. 208 (1999) 413 [hep-th/9902121] [InSPIRE].

[60] S. de Haro, S.N. Solodukhin and K. Skenderis, Holographic reconstruction of space-time and renormalization in the AdS/CFT correspondence, Commun. Math. Phys. 217 (2001) 595 [hep-th/0002230] [INSPIRE].

[61] T. Faulkner, A. Lewkowycz and J. Maldacena, Quantum corrections to holographic entanglement entropy, JHEP 11 (2013) 074 [arXiv: 1307.2892] [INSPIRE].

[62] S.A. Hartnoll, C.P. Herzog and G.T. Horowitz, Building a Holographic Superconductor, Phys. Rev. Lett. 101 (2008) 031601 [arXiv:0803.3295] [INSPIRE].

[63] J. Bhattacharya, M. Nozaki, T. Takayanagi and T. Ugajin, Thermodynamical Property of Entanglement Entropy for Excited States, Phys. Rev. Lett. 110 (2013) 091602 [arXiv: 1212.1164] [INSPIRE].

[64] D.D. Blanco, H. Casini, L.-Y. Hung and R.C. Myers, Relative Entropy and Holography, JHEP 08 (2013) 060 [arXiv: 1305.3182] [INSPIRE].

[65] A. O'Bannon, J. Probst, R. Rodgers and C.F. Uhlemann, First law of entanglement rates from holography, Phys. Rev. D 96 (2017) 066028 [arXiv: 1612.07769] [INSPIRE].

[66] H. Casini, M. Huerta and R.C. Myers, Towards a derivation of holographic entanglement entropy, JHEP 05 (2011) 036 [arXiv:1102.0440] [INSPIRE].

[67] T. Albash and C.V. Johnson, Vortex and Droplet Engineering in Holographic Superconductors, Phys. Rev. D 80 (2009) 126009 [arXiv:0906.1795] [INSPIRE].

[68] M. Montull, A. Pomarol and P.J. Silva, The Holographic Superconductor Vortex, Phys. Rev. Lett. 103 (2009) 091601 [arXiv: 0906. 2396] [INSPIRE]. 
[69] V. Keranen, E. Keski-Vakkuri, S. Nowling and K.P. Yogendran, Inhomogeneous Structures in Holographic Superfluids: II. Vortices, Phys. Rev. D 81 (2010) 126012 [arXiv:0912.4280] [INSPIRE].

[70] O. Domenech, M. Montull, A. Pomarol, A. Salvio and P.J. Silva, Emergent Gauge Fields in Holographic Superconductors, JHEP 08 (2010) 033 [arXiv: 1005.1776] [INSPIRE].

[71] N. Iqbal and H. Liu, Luttinger's Theorem, Superfluid Vortices, and Holography, Class. Quant. Grav. 29 (2012) 194004 [arXiv:1112.3671] [INSPIRE].

[72] O.J.C. Dias, G.T. Horowitz, N. Iqbal and J.E. Santos, Vortices in holographic superfluids and superconductors as conformal defects, JHEP 04 (2014) 096 [arXiv:1311.3673] [INSPIRE].

[73] K. Maeda, M. Natsuume and T. Okamura, Vortex lattice for a holographic superconductor, Phys. Rev. D 81 (2010) 026002 [arXiv:0910.4475] [InSPIRE].

[74] G. Tallarita, R. Auzzi and A. Peterson, The holographic non-abelian vortex, JHEP 03 (2019) 114 [arXiv: 1901.05814] [INSPIRE].

[75] G. Tallarita and R. Auzzi, The holographic vortex lattice using the circular cell method, JHEP 01 (2020) 056 [arXiv:1909.05932] [INSPIRE].

[76] L. Susskind, Computational Complexity and Black Hole Horizons, Fortsch. Phys. 64 (2016) 24 [Addendum ibid. 64 (2016) 44] [arXiv: 1403.5695] [INSPIRE].

[77] D. Stanford and L. Susskind, Complexity and Shock Wave Geometries, Phys. Rev. D 90 (2014) 126007 [arXiv: 1406 . 2678] [INSPIRE].

[78] A.R. Brown, D.A. Roberts, L. Susskind, B. Swingle and Y. Zhao, Holographic Complexity Equals Bulk Action?, Phys. Rev. Lett. 116 (2016) 191301 [arXiv:1509.07876] [inSPIRE].

[79] M. Moosa, Evolution of Complexity Following a Global Quench, JHEP 03 (2018) 031 [arXiv: 1711.02668] [INSPIRE].

[80] S. Chapman, H. Marrochio and R.C. Myers, Holographic complexity in Vaidya spacetimes. Part I, JHEP 06 (2018) 046 [arXiv: 1804.07410] [INSPIRE].

[81] S. Chapman, H. Marrochio and R.C. Myers, Holographic complexity in Vaidya spacetimes. Part II, JHEP 06 (2018) 114 [arXiv: 1805.07262] [INSPIRE].

[82] B. Chen, W.-M. Li, R.-Q. Yang, C.-Y. Zhang and S.-J. Zhang, Holographic subregion complexity under a thermal quench, JHEP 07 (2018) 034 [arXiv: 1803.06680] [INSPIRE].

[83] R. Auzzi, G. Nardelli, F.I. Schaposnik Massolo, G. Tallarita and N. Zenoni, On volume subregion complexity in Vaidya spacetime, JHEP 11 (2019) 098 [arXiv:1908.10832] [INSPIRE].

[84] G. Di Giulio and E. Tonni, Subsystem complexity after a global quantum quench, JHEP 05 (2021) 022 [arXiv: 2102.02764] [INSPIRE].

[85] D.S. Ageev, I.Y. Aref'eva, A.A. Bagrov and M.I. Katsnelson, Holographic local quench and effective complexity, JHEP 08 (2018) 071 [arXiv:1803.11162] [INSPIRE].

[86] D. Ageev, Holographic complexity of local quench at finite temperature, Phys. Rev. D 100 (2019) 126005 [arXiv: 1902.03632] [INSPIRE].

[87] G. Di Giulio and E. Tonni, Subsystem complexity after a local quantum quench, JHEP 08 (2021) 135 [arXiv : 2106.08282] [inSPIRE]. 